\title{
TERREMOTI DELLA VAL PADANA DEL 15-16 MAGGIO 1951
}

\author{
Caloi P. - De Panfilis M. - Di Filippo D. - Marcelli L. \\ SPADEA M. C.
}

I. Osservazioni macrosismiche. - Dalle notizie rilevate dalla Stampa e da quelle tratte dall'indagine che l'ufficio Centrale di Meteorologia ha compiuto per nostro conto, si è potuto stabilire che la zona epicentrale cadeva nel lodigiano, dove ha sviluppato una intensità valutata dal VI al VII grado della scala Mercalli.

Nelle località che seguono, l'intensità della scossa è stata appunto dal VI al VII grado. Furono inoltre osservati altri fenomeni concomitanti. Così a Codogno la scossa è stata preceduta e accompagnata da un furioso abbaiare di cani; inoltre rombi sotterranei furono avvertiti immediatamente prima della scossa. A Monza contemporaneamente alla scossa fu notato un lampeggiare in cielo accompagnato da rombi sotterranei; a Merlino oltre i rombi sotterranei fu osservato il risveglio del pollame. A Cusago il rombo fu avvertito prima della scossa. A Settala notata l'irrequietezza dei cani, bovini ed equini nonché voli di rondini. A S. Donato oltre a segni di irrequietezza ed abbaiare di cani furono osservati rombi sotterranei. L'irrequietezza degli animali prima della scossa fu notata pure a S. Giuliano Milanese. A Cremona $i$ rombi sarebbero stati susseguenti alla scossa, mentre quasi contemporanei sono stati osservati a Soncino. A Sergnano un forte rombo sotterraneo segnò l'inizio della scossa. Segni di agitazione negli animali precedettero la scossa a Caorso e Cortemaggiore. A Castel S. Giovanni, oltre a segni di irrequietezza degli animali, la scossa fu preceduta da forte rombo sotterraneo. Lo stesso avvenne a Fiorenzuola d'Adda. A Pontenure si ebbero segni di irrequietezza degli animali prima della scossa. Ad Albezzano il boato sotterraneo precedette la scossa come portato da una ventata. Cosa analoga si ebbe a Pavia e a Vistarino. Un forte boato contemporaneo alla scossa fu avvertito a Fidenza.

Altre località in cui la scossa fu sentita tra il VI ed il VII grado, 


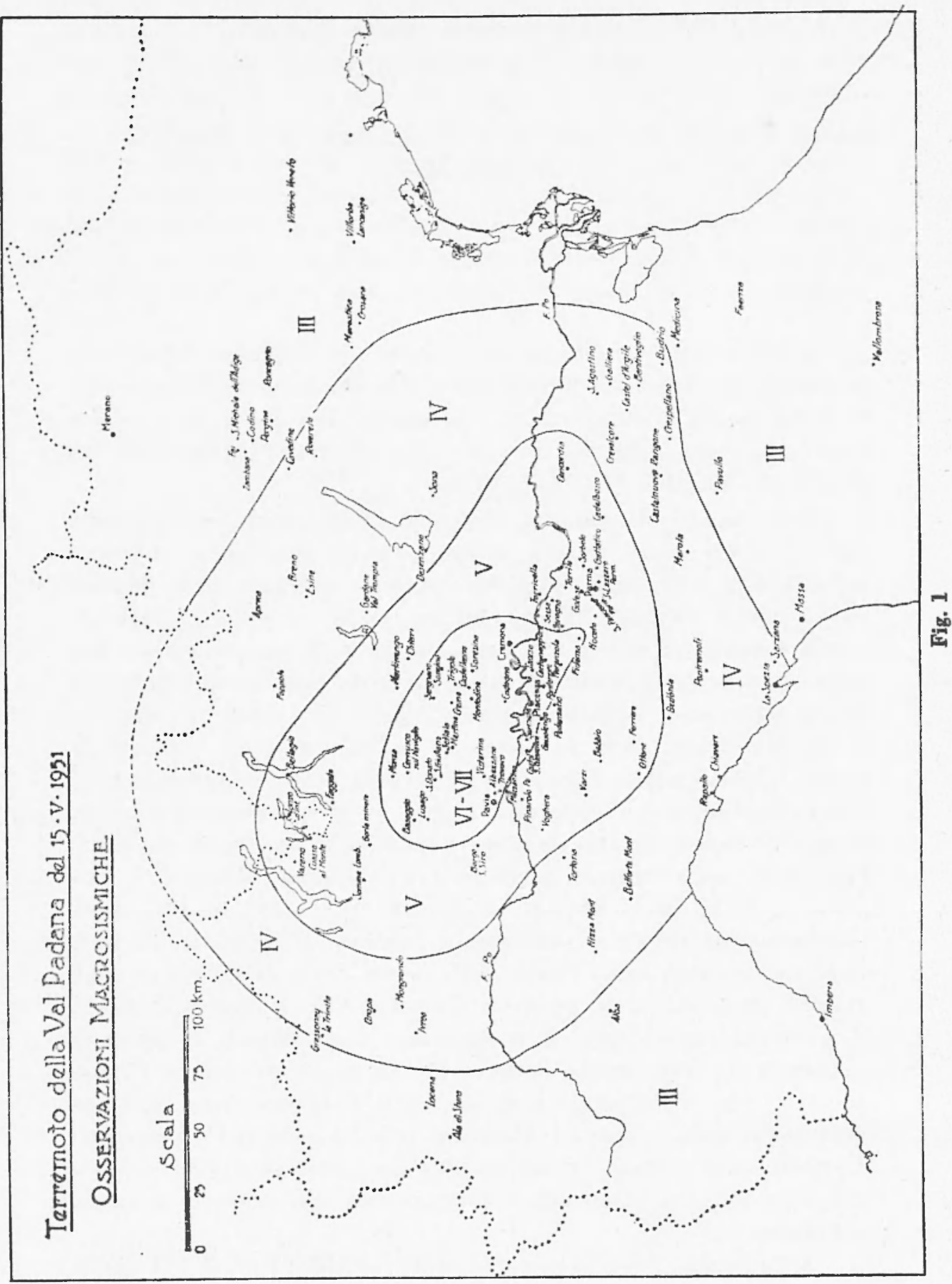


furono Cernusco sul Naviglio, Crema, Castelleone, Montodine, Sarmata, Piacenza, Travacò Siccomoro, Trigolo, Sergnano e Lusago.

La scossa di $V$ grado della scala Mercalli fu avvertita neille seguenti località: Borgo S. Siro, Pinarolo Po, Ottone, Parma, Bellagio, Gorla Minore, Cuasso al Monte, Crevalcore, Oropa, Concordia, Cadelbosco, Gattatico e Baseggio, Gossolengo, S. Lazzaro Parmense, Somma Lombardo, Gallina, Voghera.

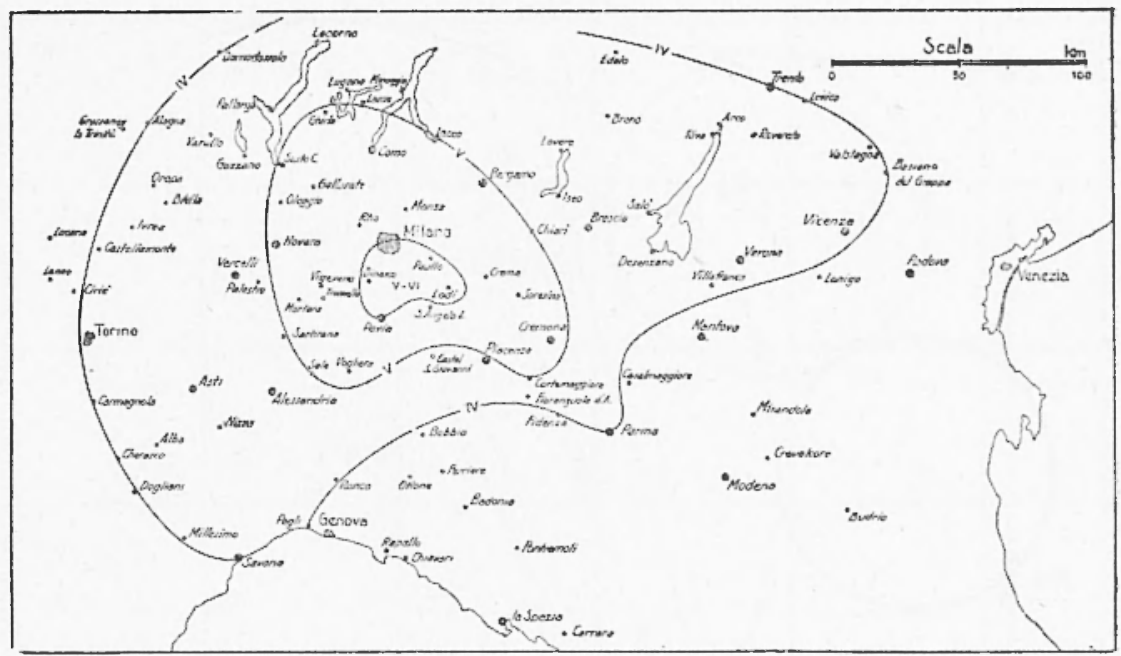

Fig. 2

Rombi sotterranei furono notati inoltre con irrequietezza di animali, nelle seguenti località: Mezzanino, Varzi, Ferriere, Podenzano, Bobbio, Soragna, Sorbolo, Noceto, Torrile, Sissa, S. Pancrazio, Faggeto Lario (con sconvolgimento delle acque del lago), Lanzo Intelvi, Varano Borghi (agitazione del lago), Chiari, Martinengo (caduta anche di una palla della torre campanaria), Torricella del Pizzo, Nizza Monferrato, Mongrando.

Di IV grado della scala Mercalli fu sentita la scossa, con rombi sotterranei e irrequietezza nelle bestie, nelle seguenti localitì: Castello d'Argile, Esine (con colpo di vento nel momento in cui la scossa era più forte), La Spezia, Aprica, S. Agostino, Cavedine. Sempre di IV grado nelle seguenti località: Bentivoglio, Budrio, Crespellano Caleno, Gardone Valtrompia, Desenzano, Breno, D'Ejola, Gressoney - La Trinité, Rapallo, Chiavari, Sarzana, Ivrea, Belforte Monferrato, Foppolo, Sona, Castelnuovo Rangone, Marola, Rovereto e Pontremoli. 
La scossa fu avvertita di III grado della scala Mercalli nelle seguenti località: Codino, S. Michele all'Adige, Fai, Pergine Valsugana, Vittorio Veneto, Locana, Ala di Stura, Merano, Marostica, Faenza, Pavullo, Medicina, Vallombrosa, Crosara e Massa Carrara. Inquietudine negli animali fu notata a Zambana, Roncegno e Imperia. A Lancenigo fu sentito inoltre un boato e come un rumore di treni in marcia. Ad Alba un rombo lievissimo nell'aria.

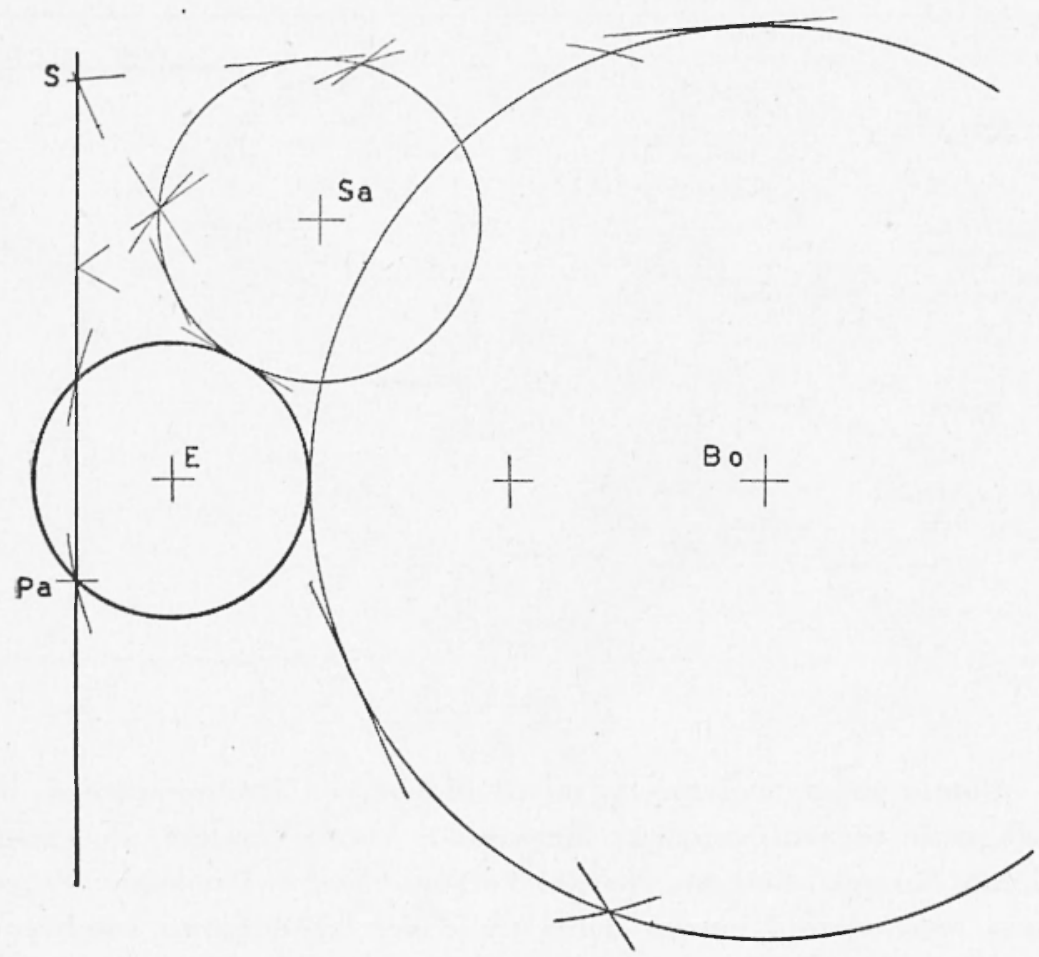

Fig. 3

Come notizie complementari, tratte dalla Stampa di Roma (e confermate sostanzialmente dalle cartoline pervenute allufficio Centrale di Meteorologia), possiamo aggiungere che la scossa è stata sentita con intensità dell'ordine del III grado Mercalli nelle due Riviere Liguri, a Verona, Asti, Vicenza, Padova, Trento, Bolzano, Feltre, Novara, Firenze, Lucca, Torino, Reggio Emilia, Imola, Vercelli e Modena. A Mantova la scossa provocò la caduta di tegole, a Brescia di calcinacci, caduta che si verificò pure a Salò, dove il lago sarebbe stato visto muoversi improvvisamente. 
TERTEMoti della yal PADANa dez 15.16 MagGio 1951

67

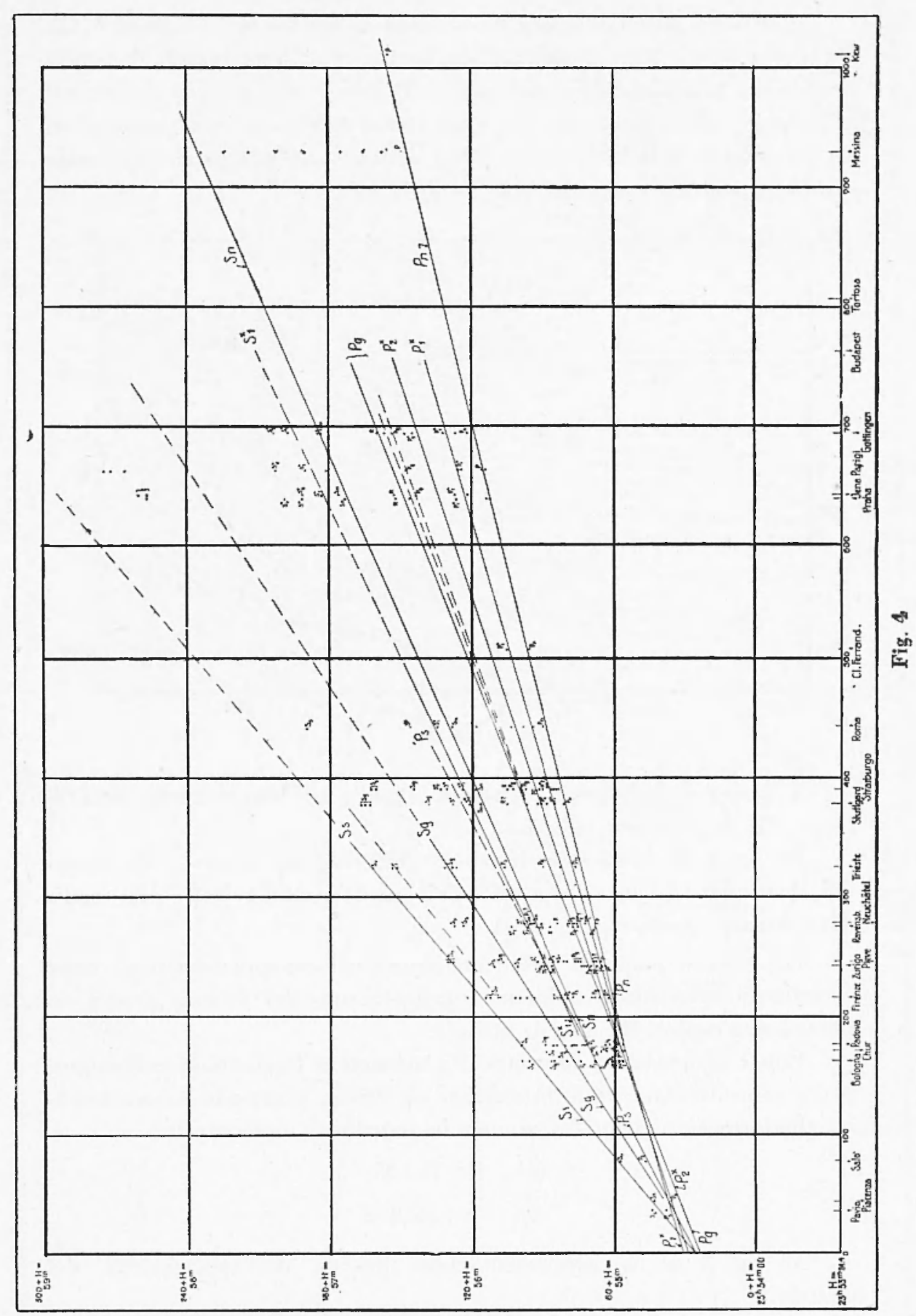


A Milano, dove la scossa fu valutata in media del VI grado della scala Mercalli, oltre a molto panico, specie nei cinematografi, la scossa determinò la caduta di cornicioni e di comignoli, nonché crepe nel selciato e lesioni piuttosto gravi in alcuni faJjoricati già danneggiati da precedenti Jombardamenti. Sia a Milano che a Venezia, la scossa sarebbe stata preceduta dal volo di piccioni.

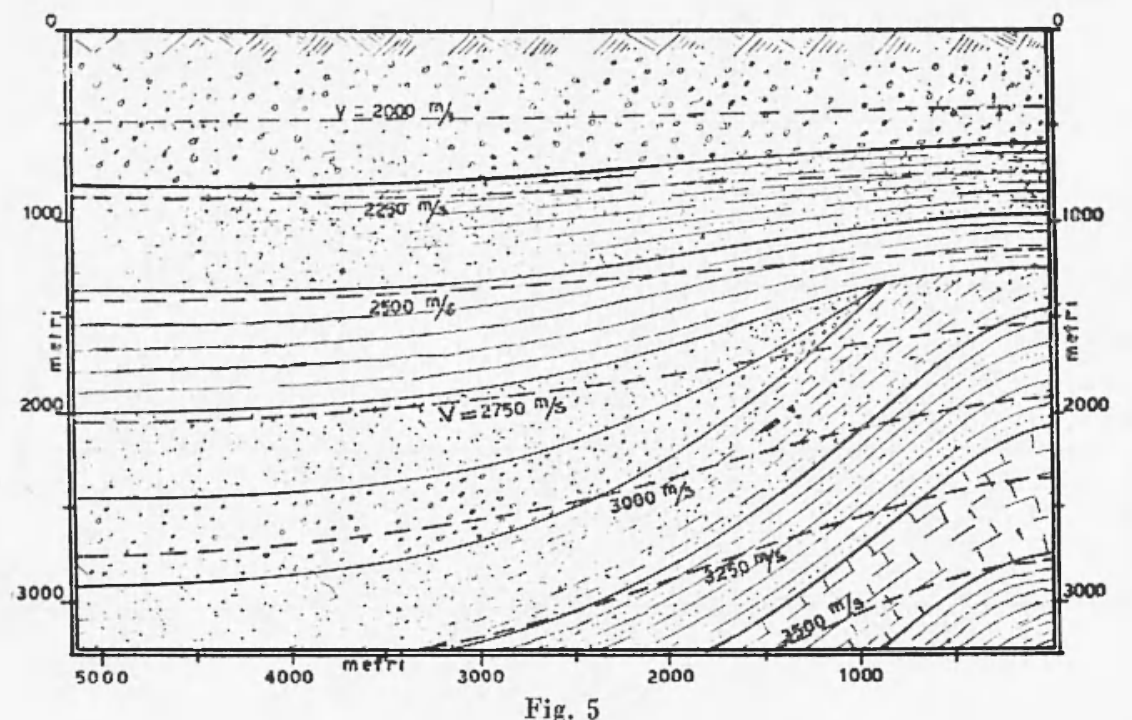

A Como e a Brumate il rombo inteso, in concomitanza con la scossa, $\mathrm{fu}$ di inusitata violenza.

La fig. 1 dà la rappresentazione delle isosiste relative alla scossa del 15 maggio. La fig. 2 riporta i dati macrosismici relativi alla scossa del mattino successivo.

II. - Allo scopo di avere un primo valore approssimativo delle coordinate epicentrali, abbiamo applicato uno dei metodi grafici, in precedenza esposti da uno di noi $\left({ }^{1}\right)$.

Con i dati relativi alle onde $P g$ ottenuti a Pavia, Salò e Bologna, presa come fondamentale la stazione di Pavia, il metodo su accennato ha condotto ai seguenti valori per le coordinate epicentrali:

$$
\begin{aligned}
& \varphi=45^{\circ} 16^{\prime} \mathrm{N} . \\
& \lambda=9^{\circ} 36^{\prime}, 8 \mathrm{E} .
\end{aligned}
$$

La fig. 3 dà la rappresentazione grafica dell'applicazione del metodo. 


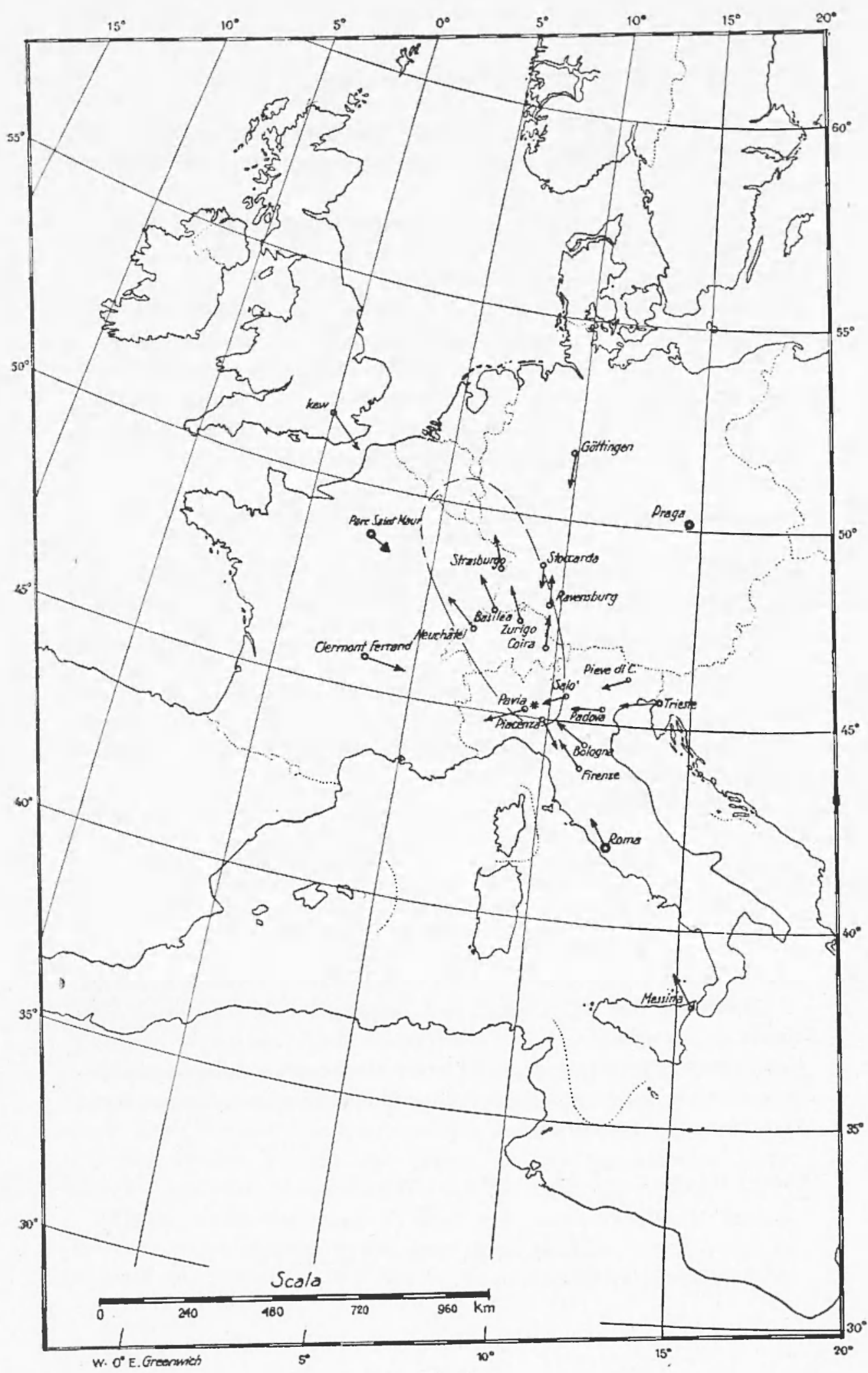

Fig. 6 
Di queste coordinate ci siamo valsi come valori approssimativi per una determinazione ulteriore, quale viene esposta nel paragrafo successivo.

III. - E stato applicato, per la determinazione delle coordinate ipocentrali e del tempo origine, un metodo che consente di ottenere contemporaneamente queste quattro grandezze $\left({ }^{2}\right)$.

Se indichiamo con $t_{0}, \lambda_{0}, \varphi_{0}$ e $h_{0}$ i valori approssimati delle incognite, con $\delta t_{\mathrm{o}}, \delta \lambda_{\mathrm{o}}, \delta \varphi_{\mathrm{o}}$ e $\delta h_{\mathrm{o}}$ le piccole correzioni da calcolare con il metodo dei minimi quadrati, per ottenere il valore più probabile delle incognite stesse e se $t_{\mathrm{n}}$ rappresenta il tempo di propagazione delle onde $P_{n}$, limitando lo sviluppo in serie ai termini di primo ordine in $\delta \lambda_{o}, \delta \varphi_{o}$, e $\bar{\delta} \tilde{n}_{0}$, supposti sufficientemente piccoli, si ha:

$$
t_{\mathrm{n}}=f\left(\lambda_{\mathrm{o}}, \varphi_{\mathrm{o}}, h_{\mathrm{o}}\right)+\frac{\partial f}{\partial \lambda_{\mathrm{o}}} \delta \lambda_{\mathrm{o}}+\frac{\partial f}{\partial \varphi_{\mathrm{o}}} \delta \varphi_{\mathrm{o}}+\frac{\partial f}{\partial h_{\mathrm{o}}} \delta h_{\mathrm{o}}
$$

In quest'ultima formula $f\left(\lambda_{u}, \varphi_{0}, h_{0}\right)$ è il tempo di propagazione delle $P_{\mathrm{n}}$ corrispondente alla distanza epicentrale della stazione considerata, quale risulta dai valori approssimati di $\lambda_{0}, \varphi_{0}$; mentre $\frac{\partial f}{\partial h_{0}}$ si ottiene dalle dromocrone, ritenendo costante $\Delta$ nel valore $\Delta_{0}$ e assegnando ad $h$ (a partire da $h_{\mathrm{o}}$ ) un incremento pari all'unità della dromocrona prescelta, che darà il corrispondente incremento positivo o negativo del tempo di tragitto.

Si prova che:

$$
\begin{gathered}
\frac{\partial f}{\partial \lambda_{0}}-\frac{\partial f}{\partial \Delta_{n}} \frac{\partial \Delta_{n}}{\partial \lambda_{0}}=-\frac{\partial f}{\partial \Delta_{r}} \cos \varphi_{0} \sin \alpha ; \\
\frac{\partial f}{\partial \varphi_{0}}=\frac{\partial f}{\partial \Delta_{n}} \frac{\partial \Delta_{n}}{\partial \varphi_{0}}=-\frac{\partial f}{\partial \Delta_{0}} \cos \alpha
\end{gathered}
$$

dove $\alpha$ indica l'azimut della stazione considerata rispetto all'epicentro.

Indicando con $\left(P_{n}\right)$ i tempi di $P_{n}$ registrati nelle stazioni di coordinate $\varphi_{n}, \lambda_{n}$, quando si faccia astrazione dagli inevitabili errori di osservazione e registrazione, detti tempi risulteranno dalla somma dell'ora iniziale del terremoto e del tempo di tragitto corrispondente, per cui varrà l'equazione

$$
\begin{aligned}
\dot{o} t_{\mathrm{o}}-\cos \varphi_{\mathrm{o}} \sin \alpha & \frac{\partial f}{\partial \Delta_{\mathrm{n}}} \bar{o} \lambda_{\mathrm{o}}-\cos \alpha \frac{\partial f}{\partial \Delta_{\mathrm{n}}} \delta \varphi_{0}+ \\
& +\frac{\partial t}{\partial h_{\mathrm{o}}} \delta h_{\mathrm{o}}=\left(\mathrm{P}_{\mathrm{p}}\right)-t_{\mathrm{o}}-f\left(\lambda_{\mathrm{o}}, \varphi_{\mathrm{o}}, h_{\mathrm{o}}\right)
\end{aligned}
$$


che è l'equazione di condizione relativa alla stazione considerata, di distanza epicentrale $\Delta_{\mathrm{n}}$; il secondo membro è costituito da quantità note.

La [2] naturalmente va ripetuta per tutte le stazioni ed il sistema che così si ottiene risolto con $i$ minimi quadrati.

Una prima applicazione della [2] ̀̀ fatta con i seguenti dati ap-

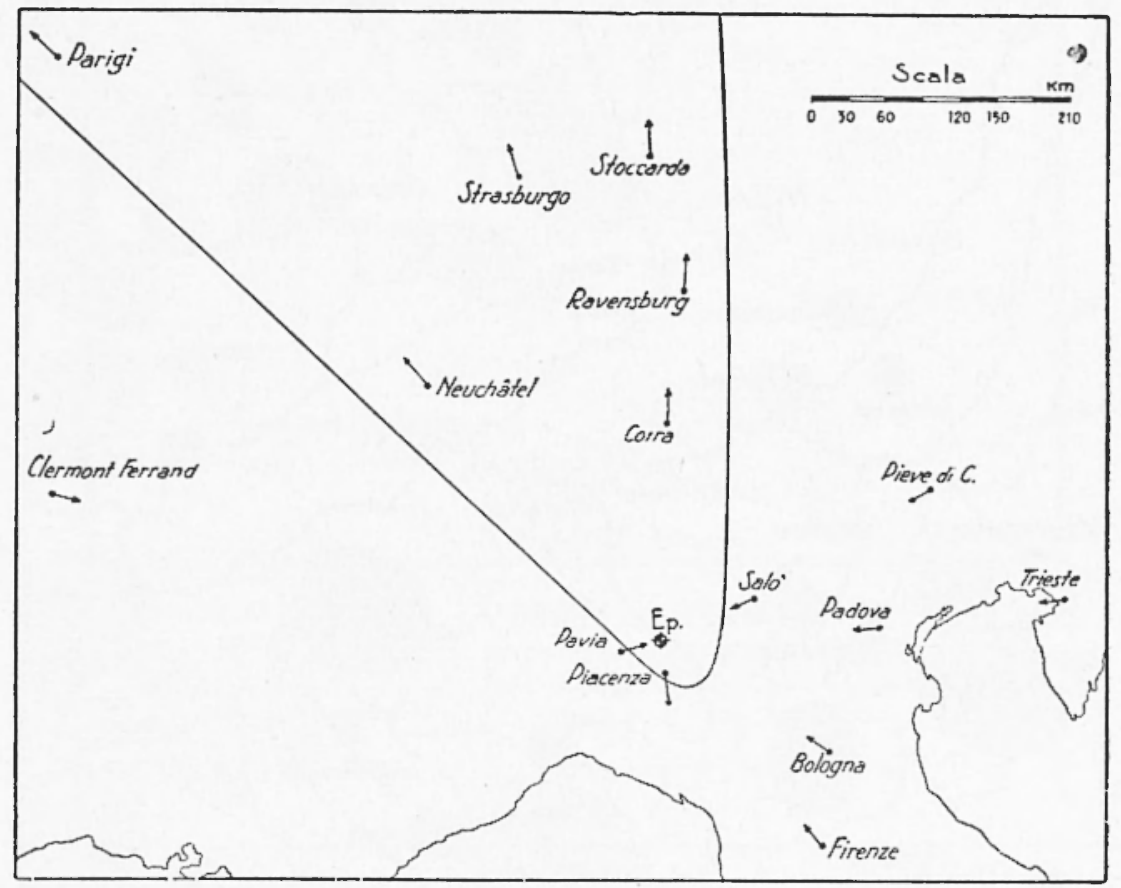

Fig. 6

prossimativi: $\quad \lambda_{0}=9^{\circ} 36^{\prime}, 8 \mathrm{E} ; \varphi_{\mathrm{o}}=45^{\circ} 16^{\prime} \mathrm{N} ; h_{0}=5 \mathrm{~km} ; t_{0}=$ $22^{\mathrm{h}} 54^{\mathrm{m}} 24^{\mathrm{s}}$.

Le stazioni di cui ci siamo serviti furono Coira, Zurigo, Neuchâtel, Stoccarda, Strasburgo, Roma, Praga, Messina. La Tabella I contiene alcuni degli elementi che hanno servito per la risoluzione, con il metodo dei minimi quadrati del sistema di otto equazioni analoghe alla [2], applicata ad ognuna delle otto stazioni considerate.

Per il calcolo dei tempi di tragitto ci siamo valsi della dromocrona relativa al terremoto del Gran Sasso d'Italia, per il quale fu calcolata una profondità di $5 \mathrm{Km}$ ca. La scelta è pure giustificata dal 




Fig. 8 
fatto che, in lavori precedenti, è stato provato che le velocità di propagazione negli strati superficiali della Val Padana hanno stretta analogia con quelle ottenute per l'Italia centrale.

L'applicazione del metodo ha portato ai seguenti valori più pro-

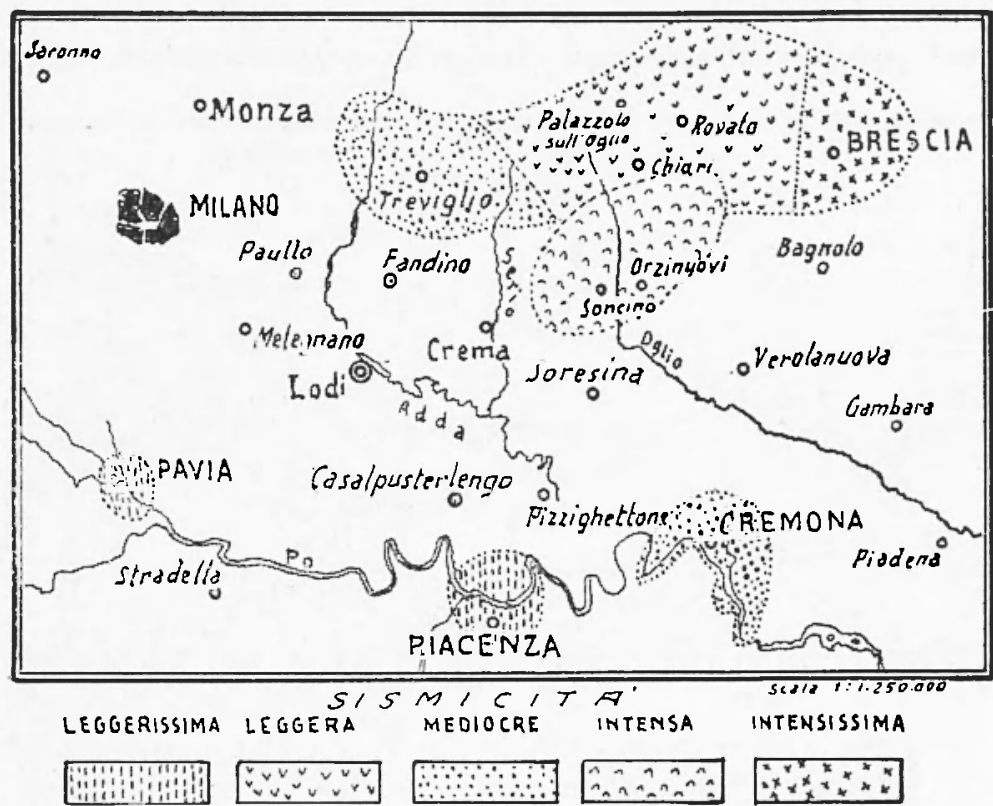

Fig. 9

Tabella I

\begin{tabular}{|c|c|c|c|c|c|c|}
\hline STAZIONI & $\Delta$ & $\operatorname{sen} \alpha$ & $\alpha$ & $\cos \alpha$ & 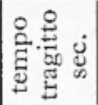 & tempo calcol. \\
\hline Coira & $1^{\circ} .584$ & -.0 .033 & $358^{\circ} .06^{\prime} .11^{\prime \prime}$ & -0.999 & $31^{s} .9$ & $22^{\mathrm{h}} 54 \mathrm{~m} 55 \mathrm{~s} .9$ \\
\hline Zurigo & $2^{\circ} .218$ & -0.316 & $341^{\circ} .36^{\prime} .32^{\prime \prime}$ & $\begin{array}{r}0.949 \\
+\quad 0.9\end{array}$ & 40.5 & $\begin{array}{lll}55 & 04.5\end{array}$ \\
\hline Neuchâtel & 20.526 & -0.717 & $314^{\circ} .11^{\prime} .30^{\prime \prime}$ & $\begin{array}{l}0.697 \\
+\quad 0.07\end{array}$ & 44.7 & 08.7 \\
\hline Stoccarda & $3^{\circ} .516$ & --0.079 & $355^{\circ} .28^{\prime} .54^{\prime \prime}$ & $\begin{array}{r}0.997 \\
+0 .\end{array}$ & 58.1 & 22.1 \\
\hline Strasburgo & 30.550 & --0.345 & $239^{\circ} .50^{\prime} .38^{\prime \prime}$ & $\begin{array}{r}+ \\
+\end{array}$ & 58.6 & 22.6 \\
\hline Roma & 30.965 & +0.545 & $147^{\circ} .00^{\prime} .11^{\prime \prime}$ & -0.839 & 64.2 & 28.2 \\
\hline Praga & $5^{0} .794$ & $\begin{array}{r}0.534 \\
+\end{array}$ & $32^{\circ} .16^{\prime} .50^{\prime \prime}$ & +0.845 & 89.0 & 53.0 \\
\hline Messina & $8^{\circ} .340$ & +0.561 & $145^{\circ} .53^{\prime} .00^{\prime \prime}$ & -0.828 & 123.5 & $\begin{array}{lll}56 & 27.5\end{array}$ \\
\hline
\end{tabular}

babili delle incognite: $\bar{o} \lambda_{0}=-11^{\prime}, 13 \pm 4^{\prime}, 2 ; \dot{\partial} \varphi_{0}=+3^{\prime}, 95 \pm 1^{\prime}, 8$; $\delta t_{0}=0^{\star}, 3+0^{4}, 3 ; \delta h_{\mathrm{n}}=0$

Il controllo dei calcoli, ha portato ai risultati; $[v v]=2,35075$; [11.4] $=2,35075$ a conferma dell'esattezza dei risultati. 
Tenendo conto dei valori di partenza si ha pertanto $\lambda_{\mathrm{E}}=9^{\prime \prime} 25^{\prime}, 7$ $\left.\pm 4^{\prime}, 2 ; \varphi_{E}=45^{\circ} 20^{\prime}\right\rfloor_{-}^{\prime}, 8 ; t_{0}=22^{\mathrm{h}} 54^{\mathrm{m}} 24^{\mathrm{s}}, 3+0^{\mathrm{s}}, 3$. La profondità ipocentrale risulta dell'ordine di $5 \mathrm{Km}$.

IV. - Un valore approssimativo per le coordinate epicentrali ̀̀ stato ottenuto con un terzo metodo che si vale dei dati di quattro stazioni prossime all'epicentro. Assegnate quattro stazioni, una delle

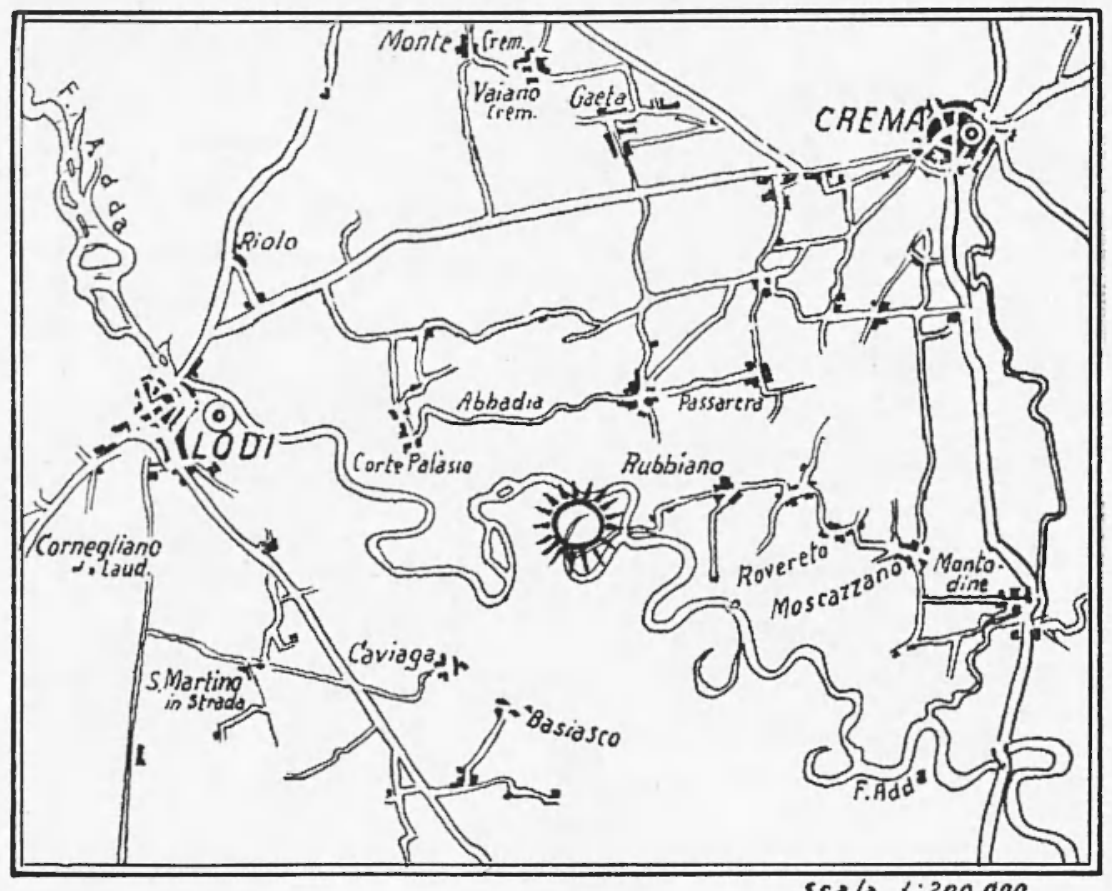

Fig. 10

quali, la più vicina, scelta come fondamentale, conosciuti i tempi di registrazione di un tipo di onda longitudinale diretta nelle stazioni $0_{1}, 0_{2}, 0_{3}, 0_{4}$, indichiamo con $\delta_{1}, \delta_{2}, \delta_{3}$ le differenze fra i tempi di registrazione dell'onda longitudinale considerata nelle tre stazioni più lontane ed il tempo di registrazione della stessa nella stazione fondamentale. Nel caso di un epicentro prossimo alla superficie, le tre circonferenze di raggi v. $\delta_{1}$, v. $\delta_{2}$, v. $\delta_{3}$ e centri in $0_{2}, 0_{3}, 0_{4}$, dovranno risultare tangenti esternamente ad una circonferenza che ha per centro lepicentro.

Di tale problema è stata data una risoluzione geometrica ed una analitica. Qui ci varremo della soluzione analitica $\left({ }^{3}\right)$. 


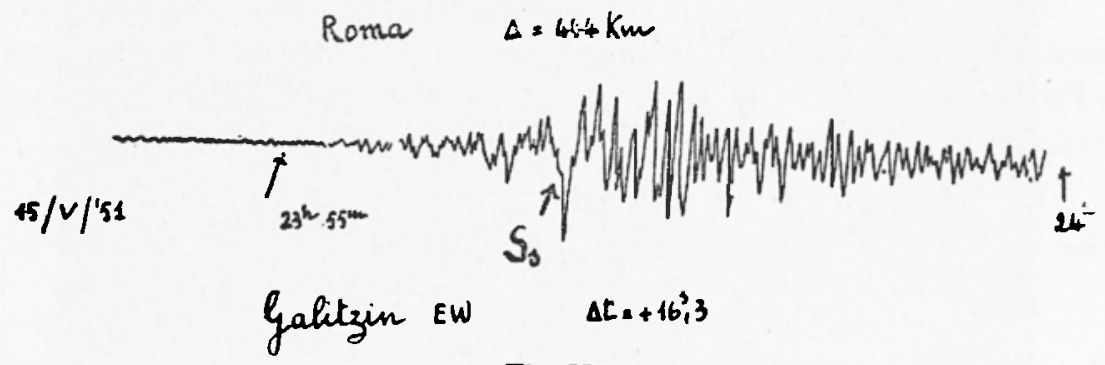

Fig. 11

Poniamo le origini delle coordinate al centro della circonferenza $0_{2}$ ed indichiamo con $x, y$ le coordinate del punto di contatto di $0_{2}$ con $E$ - circonferenza avente per centro l'epicentro - Se $r, r^{\prime}, r^{\prime \prime}$ sono i raggi delle tre circonferenze $0_{2}, 0_{3}, 0_{4}, R$ il raggio della circonferenza $E$ e se $X, Y$, sono le coordinate del centro di $E$, si ha

$$
X=\frac{x(R+r)}{r} \quad, \quad Y=\frac{y(R+r)}{r} .
$$

Indichiamo con $\alpha^{\prime}, \beta^{\prime} ; \alpha^{\prime \prime}, \beta^{\prime \prime}$, le coordinate dei centri di $0_{3}, 0_{4}$, rispetto all'origine (centro di $0_{2}$ ).

Fatte le posizioni

si ottiene

$$
\begin{gathered}
\rho^{\prime 2}=\alpha^{\prime 2}+\beta^{\prime 2}-\left(r-r^{\prime}\right)^{2} ; \rho^{\prime 2}=\alpha^{\prime \prime 2}+\beta^{\prime \prime 2}-\left(r-r^{\prime \prime}\right)^{2} \\
\varepsilon_{1}=\rho^{\prime \prime 2} \alpha^{\prime}-\rho^{\prime 2} \alpha^{\prime \prime} ; \varepsilon_{2}=\rho^{\prime \prime 2} \beta^{\prime}-\rho^{\prime 2} \beta^{\prime \prime} \\
\sigma=\rho^{\prime 2}\left(r^{\prime \prime}-r\right)-\rho^{\prime \prime 2}\left(r^{\prime}-r\right) \\
\chi=\sqrt{\varepsilon_{1}^{2}+\varepsilon_{2}{ }^{2}-\sigma^{2}}
\end{gathered}
$$

$$
x=r \frac{\sigma \varepsilon_{1} \mp \chi \varepsilon_{2}}{\varepsilon_{1}^{2}+\varepsilon_{2}^{2}} \quad, \quad y=r \frac{\sigma \varepsilon_{2} \pm \chi \varepsilon_{1}}{\varepsilon_{1}^{2}+\varepsilon_{2}^{2}} .
$$

È inoltre

$$
\begin{aligned}
R= & \frac{r}{4}\left[\frac{r^{2}-r^{\prime 2}+\alpha^{\prime 2}+\beta^{\prime 2}-2 \alpha^{\prime} x-2 \beta^{\prime} y}{\dot{\alpha}^{\prime} x+\beta^{\prime} y+r\left(r^{\prime}-r\right)}+\right. \\
& \left.+\frac{r^{2}-r^{\prime \prime 2}+\alpha^{\prime \prime 2}+\beta^{\prime \prime 2}-2 \alpha^{\prime \prime} x-2 \beta^{\prime \prime} y}{\alpha^{\prime \prime} x+\beta^{\prime \prime} y+r\left(r^{\prime \prime}-r\right)}\right] .
\end{aligned}
$$

Sostituite le [4] nella [5] si ottengono due valori per $R$.

Le [3] danno allora due coppie di valori per $X, Y$. É facile stabilire quale di queste coppie fornisca le coordinate dell'epicentro. 
Nel nostro caso si è fatto uso dei tempi di registrazione delle onde longitudinali proprie dei sedimenti, ottenuti a Pavia, Salò, Bologna e Padova. Come si vedrà più avanti, la velocitá media di propagazione è risultata di $\mathrm{Km} 3,8$ al sec.

I dati sono contenuti nell'unita tahellina:

\begin{tabular}{|c|c|c|c|c|}
\hline & $P_{u}$ & $\tilde{\partial} P_{t}$ & $\tilde{c} \Delta$ & \\
\hline Pavia & $23.54 .37,9$ & & & \\
\hline Salò & 45,6 & 7,7 & $29,26(\mathrm{r})$ & $v_{P_{n}}=3,8 \mathrm{~km} / \mathrm{sec}$ \\
\hline Bologna & 55.08 & 30,1 & $114,38\left(\mathrm{r}^{\prime}\right)$ & $\mathbf{r}^{\prime}-\mathbf{r}=85,12$ \\
\hline Pádova & 12 & 34,1 & $129,58\left(r^{\prime \prime}\right)$ & $r^{\prime \prime}-r=100,32$ \\
\hline
\end{tabular}

Presa come origine delle coordinate Salò (asse delle ordinate, il meridiano per Salò; asse delle ascisse, il cerchio massimo a $90^{\circ}$, in direzione orientale), con metodi della geodesia, si trova, note le coordinate geografiche di Bologna e Padova, che le coordinate ortogonali di queste ultime stazioni (centri delle circonferenze $0_{3}, 0_{4}$ ) sono:

$$
\begin{aligned}
\alpha^{\prime} & =63,95 \mathrm{Km}, \mathrm{y}^{\circ}=-124,05 \mathrm{Km} \\
\alpha^{\prime \prime} & =104,94 \mathrm{Km}, \beta^{\prime \prime}=-22,18 \mathrm{Km} .
\end{aligned}
$$

L'applicazione delle [4], conduce ai seguenti valori per le $x, y$

$$
x=\left\{\begin{array}{l}
-27,83 \\
-26,09
\end{array} \quad, \quad y=i-9,045,\right.
$$

Dalla [5] calcoliamo $R$ con la prima coppia di valori per $x, y$. Si ottiene $R=68,45$.

Consegue $X=-92,95 \mathrm{Km} ; Y=-30,21 \mathrm{Km}$.

Queste ultime sono le coordinate ortogonali dell'epicentro rispetto a Salò. Trasformate in geografiche, l'epicentro risulta individuato dal punto

$$
\emptyset=45^{\circ} 19^{\prime}, 8 \cdot N ; \lambda=9^{\circ} 20^{\prime} E \mathrm{Gr} \text {. }
$$

L'epicentro così ottenuto coincide praticamente con quello cui siamo pervenuti applicando il metodo relativo ai tempi di registrazione delle onde $P_{n}$.

Va osservato che il valore di $3,8 \mathrm{Km} / \mathrm{sec}$ è forse un po' eccessivo per quanto si riferisce a Pavia, data la sua vicinanza all'epicentro e la maggiore influenza che sui tempi di propagazione hanno le strati- 


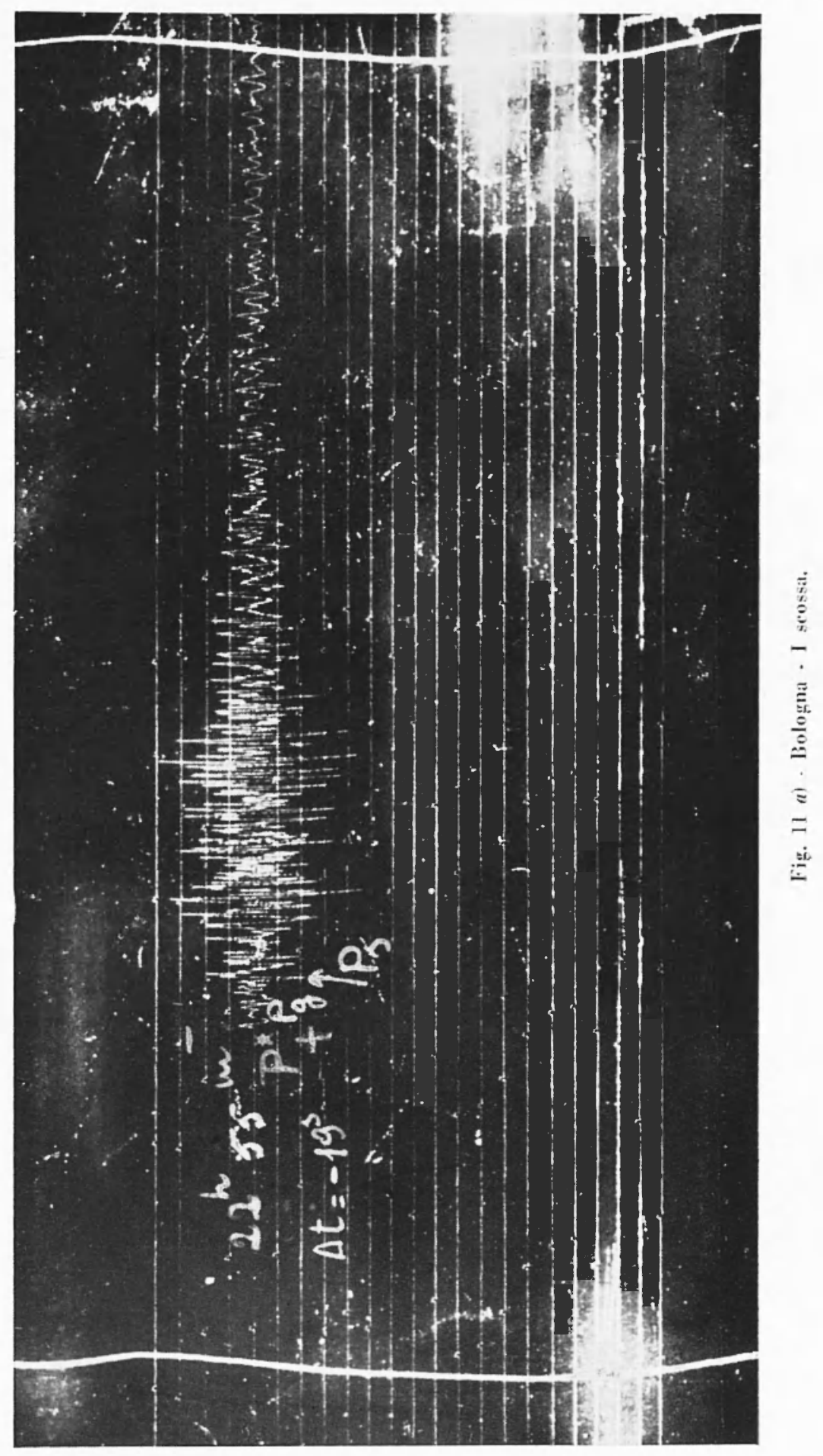




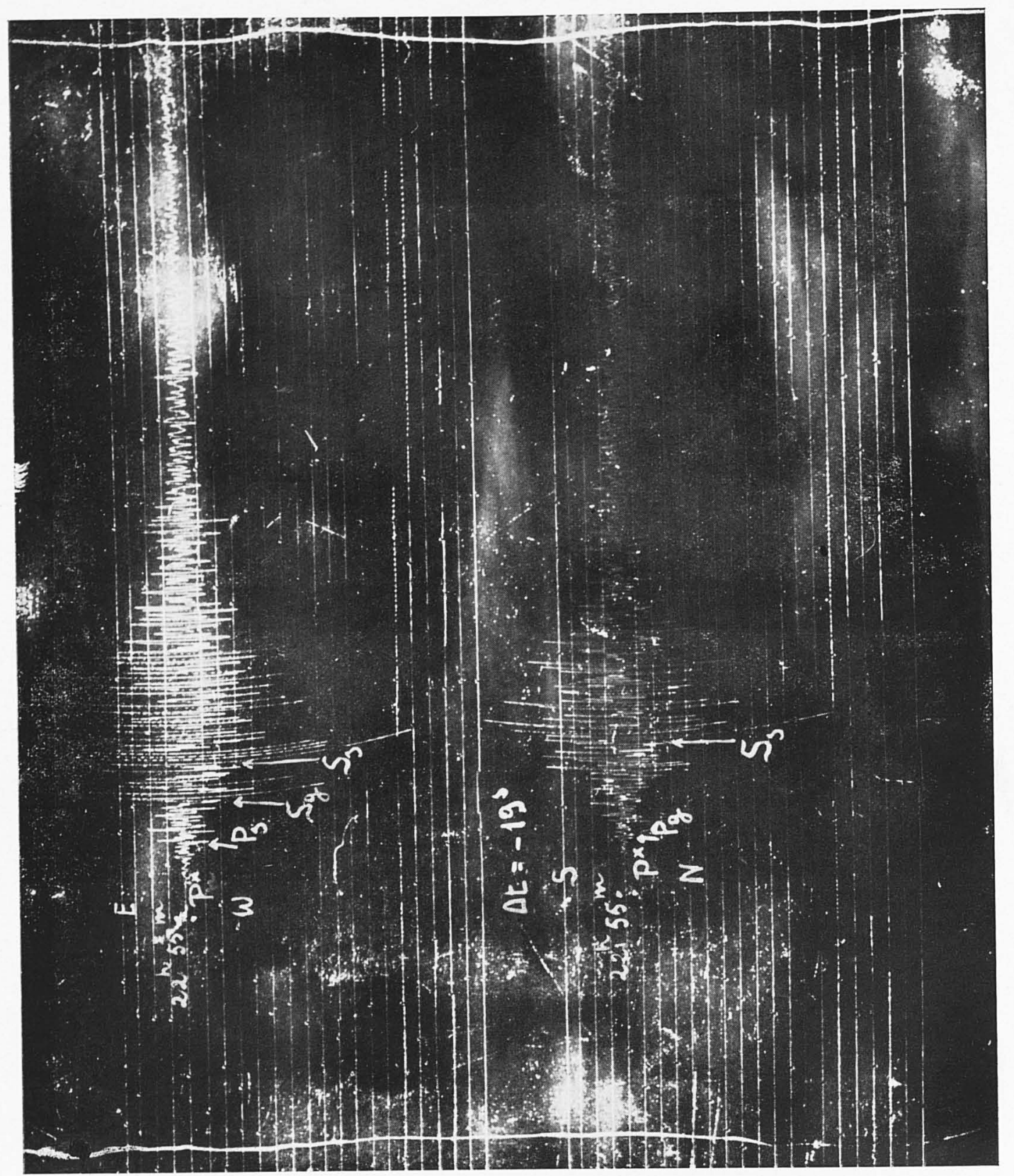


ficazioni più superficiali dei sedimenti. Questo fatto porta ad un probabile lieve spostamento dell'epicentro verso occidente.

V. - Per una più precisa determinazione dell'epicentro, abbiamo ritenuto opportuno ricorrere ad un metodo che consentisse di prescindere dagli eventuali errori nei tempi di registrazione delle singole onde dirette. Come si è già osservato, l'epicentro cade in una zona caratterizzata da strati sedimentari di notevole spessore. Allo scopo di avere ridotta al minimo l'influenza di detti strati sulla propagazione delle onde sismiche $P_{g}$ ed $S_{g}$ abbiamo scelto i tempi relativi a queste onde ottenuti in stazioni sismiche situate oltre la val Padana, dentro la zona alpina.

Il metodo in questione è basato sulla conoscenza delle difierenze dei tempi di tragitto delle onde $S_{\mathrm{g}}$ e $P_{\mathrm{k}}$ ( $\left.^{4}\right)$. Se $k=\frac{v_{1} \cdot v_{2}}{v_{1}-v_{2}}$, dove $v_{1}, v_{2}$ sono le velocità di propagazione delle onde longitudinalí e trasversali dirette; se $x_{0}, y_{0}, z_{0}$, sono le coordinate dell'ipocentro e $x_{i}, y_{i}, z_{i,}$ le analoghe coordinate di una generica stazione $S_{\text {i }}$, riferite alla medesima origine, trascurando il valore di $z_{i}$, possiamo scrivere

$$
\Phi_{\mathrm{i}}=\frac{1}{k} V\left(\overline{\left.x_{\mathrm{i}}-x_{\mathrm{o}}\right)^{2}+\left(y_{\mathrm{i}}-y_{\mathrm{o}}\right)^{2}+z_{\mathrm{o}}{ }^{2}}-\left(t_{\mathrm{z}}-t_{1}\right)_{\mathrm{i}}, \quad(i=1,2, \ldots n)\right.
$$

dove $t_{1}, t$. indicano i tempi di registrazione delle onde $P_{g}, S_{\mathrm{g}}$ in una stazione assegnata.

Deve essere

$$
\Phi_{\mathrm{i}}=\Phi_{\mathrm{i}}\left(x_{\mathrm{o}}, y_{\mathrm{o}}, z_{\mathrm{o}}, k\right)=0
$$

Se il numero delle equazioni supera quello delle incognite e se $\left(x_{0}\right)$, $\left(y_{0}\right),\left(z_{0}\right),(k)$ è un sistema di valori approssimati delle incognite stesse, possiamo pord $\therefore-\left(x_{0}\right)+x ; y_{0}=\left(y_{0}\right)+y ; z_{0}=\left(z_{0}\right)+z ; k=(k)+k_{1}$, dove $x, y, z, k_{1}$, rappresentano le correzioni incognite.

Si ha, limitando lo sviluppo al primo termine,

$$
\Phi_{1}=\Phi_{i}(0)+\frac{\partial \Phi_{i}(n)}{\partial\left(x_{0}\right)} x+\frac{\partial \Phi_{i}(0)}{\partial\left(y_{0}\right)} y+\frac{\partial \Phi \cdot(0)}{\partial\left(z_{0}\right)} z+\frac{\partial \Phi_{i}(0)}{\partial(k)} k_{1},
$$

dove $\Phi_{\mathrm{i}}(0)$ e $\mathrm{i}$ coefficienti di $x, y, z, k_{1}$, hanno valori facilmente determinabili con i dati di partenza.

Notizie più dettagliate sull'applicazione del metodo, la determinazione degli errori medi delle incognite, ecc. si trovano nella nota espositiva del procedimento di calcolo. 
Per l'applicazione del metodo nel caso in esame, ci siamo valsi dei dati di cinque stazioni: Coira, Zurigo, Neuchâtel, Trieste, Pieve di Cadore.

La trasformazione delle coordinate geografiche delle varie stazioni in coordinate ortogonali - trasformazione eseguita con metodi geodetici, riassunti in una nota precedente - fu fatta rispetto al punto origine $\varphi_{0}=45^{\circ}, 3 \mathrm{~N} ; \lambda_{\circ}=9^{\circ}, 6 \mathrm{E} \mathrm{Gr}$.

L'unita tabella contiene le coordinate geografiche e ortogonali delle varie stazioni, assieme ai valori $\left(t_{2}-t_{1}\right)_{\mathrm{i}}$ delle differenze $S_{\mathrm{g}}-P_{\mathrm{g}}$, osservate nelle stazioni stesse.

\section{Tabella I}

\begin{tabular}{|l|l|l|c|}
\hline \multicolumn{1}{|c|}{ STAZIONI } & \multicolumn{1}{|c|}{ Coordin. Geograf. } & $\begin{array}{c}\text { Coord. Ort. } \\
\text { Km }\end{array}$ & $\begin{array}{c}\mathrm{t}_{2}-\mathrm{t}_{1} \\
\text { sec. }\end{array}$ \\
\cline { 2 - 4 } & $\begin{array}{l}46^{\circ} .51^{\prime} \mathrm{N} ; 9^{\circ} .32^{\prime}, 2 \mathrm{E} \\
\text { Coira }\end{array}$ & $-4,81 ;+172,24$ & 22,0 \\
Zurigo & $47^{\circ} .22^{\prime}, 1 \mathrm{~N} ; 8^{0} .34^{\prime}, 8 \mathrm{E}$ & $-76,55 ;+230,35$ & 31,1 \\
Neuchâtel & $46^{0} .59^{\prime}, 85 \mathrm{~N} ; 6^{0} .57^{\prime}, 45 \mathrm{E}$ & $-200,24 ;+192,01$ & 35,7 \\
Trieste & $45^{\circ} .38^{\prime}, 6 \mathrm{~N} ; 13^{\circ} .45^{\prime}, 1 \mathrm{E}$ & $+322,40 ;+46,51$ & 41,5 \\
Pieve di Cadore & $46^{\circ} .24^{\prime}, 6 \mathrm{~N} ; 12^{\circ} .22^{\prime}, 2 \mathrm{E}$ & $+212,21 ;+127,07$ & 31,7 \\
\hline
\end{tabular}

Una prima applicazione del metodo fu fatta con $i$ seguenti dati iniziali : $\left(x_{0}\right)=0 ;\left(y_{0}\right)=0 ;\left(z_{0}\right)=5 ;(k)=7,8$.

Questa applicazione ha portato ad una forte correzione negativa per la profondità. Ciò significa che la profondità ipocentrale va considerata praticamente nulla.

Naturalmente l'annullarsi della profondità ipocentrale va intesa nel senso che l'ipocentro stesso è da ritenersi ai limiti esterni dello strato del granito; e precisamente dove questo confina con gli strati sedimentari costituiti dalla coltre della Val Padana.

Le correzioni in $k, x, y$, furono le seguenti:

$$
k_{1}=-0,014 ; x=+0,91 ; y=-0,066 \text {. }
$$

Pertanto sono stati ripetuti i calcoli con i seguenti dati:

$$
\left(x_{\mathrm{o}}\right)=+0,91 ;\left(y_{0}\right)=-0,07 ;\left(z_{0}\right)=0 ;(k)=7,79,
$$

che hanno condotto ai risultati qui riportati:

$$
x=+0,174 ; y=+0,675 ; k_{1}=-0,000516 \text {. }
$$

Gli scarti per le singole stazioni furono:

$$
\begin{gathered}
v_{1}=+0,0456 ; v_{2}=+0,0270 ; v_{3}=-0,0413 \\
v_{4}=+0,0682 ; v_{\overline{\mathbf{s}}}=-0,1018
\end{gathered}
$$


La verifica dei calcoli fu ottenuta determinando il valore di [11.3] e di $[v v]$. Si ebbe, a conferma dei risultati, $[11.3]=[v v]=0,01952$.

Determinati grli errori medi dei valori più probabili delle incognite, siamo pervenuti ai seguenti risultati finali:

$$
x_{0}=+1,08+0,65 ; y_{0}=+0,61 ; 1,02 ; k=7,79+0,02 \text {. }
$$

Trasformate le coordinate ortogonali in geografiche, si ottengono in definitiva, per le coordinate epicentrali, $i$ seguenti valori:

$$
\varphi=45^{\circ} 18^{\prime}, 33 \pm 0^{\prime} 55 \mathrm{~N} ; \lambda=9^{\circ} 36^{\prime}, 83 \pm 0^{\prime} 50 \mathrm{E} . \mathrm{Gr} \text {. }
$$

VI. -- Come era da prevedere, data la particolare posizione dell'epicentro, la propagazione delle onde longitudinali e trasversali dirette (onde $P_{k}$ ed $S_{g}$ ) risente in modo sensibile della conseguente situazione geologica. Ciò ha reso alquanto più difficile dell'ordinario l'interpretazione dei sismogrammi di cui la Tabella II dà un quadro complessivo.

Le onde $P_{\underline{k}}$ hanno tempi di tragitto che non consentono la determinazione di una unica dromocrona rettilinea. Pertanto abbiamo suddiviso le stazioni in due gruppi, tralasciando i dati che si riferiscono alla Val Padana propriamente detta. In un primo gruppo abbiamo considerato le stazioni dei cui dati ci siamo serviti per determinare l'epicentro. Con i tempi di registrazione per le onde $P_{k}$ ottenuti a Coira, Zurigo, Pieve di Cadore, Neuchâtel e Trieste siamo pervenuti alla seguente equazione della dromocrona più probalsile per le onde $P_{x}$ :

$$
\iota=0,1968 \Delta-0,64
$$

che può anche scriversi

$$
t_{\mathrm{H}}=\frac{\Delta}{5,08}-0,64
$$

I calcoli sono stati eseguiti scegliendo come ora origine provvisoria $54^{\mathrm{m}} 25^{\mathrm{s}}$. Albliamo visto che l'ipocentro cade ai margini esterni dello strato del "granito ). Il tempo dedotto dalla dromocrona delle onde $P_{K}$ può quindi essere scelto come tempo origine effettivo. Perciò avremo:

$$
t_{\mathrm{H}}=23^{\mathrm{n}} 54^{\mathrm{m}} 24^{\mathrm{s}}, 4 \text {. }
$$

Gli scarti dei tempi di registrazione dalla dromocrona sono sensibili (Coira, + 1,0 - tempi osservati meno tempi calcolati -; Zurigo, - 2,4; Pieve di Cadore, $+1,7$; Neuchâtel, $-2,1$; Trieste, $+1,8$ ). 
Ciò si spiega con il fatto che nella zona della Val Padana la transizione fra gli strati sedimentari consolidati ed il sottostante "granito " avviene certamente in modo graduale e nello stesso tempo saltuario, cosi da determinare inegruali rallentamenti nelle diverse direzioni. Con questo si spiega pure il fatto che la media delle velocità per le onde $P_{b}$ è risultata, nella zona interessata dalle stazioni prese in considerazione, molto al di sotto dei valori in precedenza calcolati per regioni limitrofe.

Avviene infatti che per le stazioni sismiche più lontane (Ravensburg, Stoccarda, Strasburgo, ecc.) la media velocità di propagazione delle onde $P_{F}$ tende a crescere con la distanza, come è provato dalla dromocroma (fig. 4).

Per le onde trasversali dirette $S_{\mathrm{g}}$ si è seguito un procedimento analogo a quello applicato per le $P_{\mathrm{g}}$. Con i tempi di registrazione ottenuti a Coira, Zurigo, Pieve di Cadore, Neuchatel, e Trieste e con riferimento al tempo origine provvisorio $54^{\mathrm{m}} 25^{\mathrm{s}}$, abljiamo ottenuto la seguente equazione per la dromocrona più probabile relativa alle onde $S_{k}$ :

$$
t=0,3249 \Delta-0,58 \text {. }
$$

Ciò porta ad una velocità pari a $3,08 \mathrm{Km} / \mathrm{sec}$ per le onde $S_{\mathrm{g}}$.

Come si vede, il tempo origine che ne consegue coincide con quello ottenuto con le onde $P_{g}$.

Tenuto conto dei valori avuti per la velocità delle onde $P_{g}$ ed $S_{g}$ si ottiene per il parametro $k$ il valore 7,82 , in buonissimo accordo quindi (fino quasi alla coincidenza) con il valore ottenuto per detto parametro nell'applicazione del metodo che sfrutta $i$ tempi di tragitto delle onde $S_{\mathrm{g}}$ e $P_{\mathrm{g}}$ nella determinazione delle coordinate ipocentrali.

Come per le onde $P_{g}$, anche per le $S_{g}$ naturalmente le differenze fra i valori osservati e quelli calcolati sono sensibili :

Coira, $+1,0 ;$ Zurigo, - 2,4; Pieve di Cadore, $+1,8$; Neuchâtel, $-2,1 ;$ Trieste, $+1,7$.

Sul motivo di questi scarti si è già detto parlando delle onde $P_{\text {rg }}$.

VII. - Una delle parti più interessanti del lavoro oggetto di questa pubblicazione, è quella che si riferisce alle stratificazioni che precedono il così detto strato del "granito " e che noi indicheremo con il titolo generico di sedimenti. Gli strati alluvionali hanno indubbiamente in Val Padana spessori notevoli, anche se variabili da luogo a luogo. La posizione particolare dell'epicentro, il fatto che l'origine 


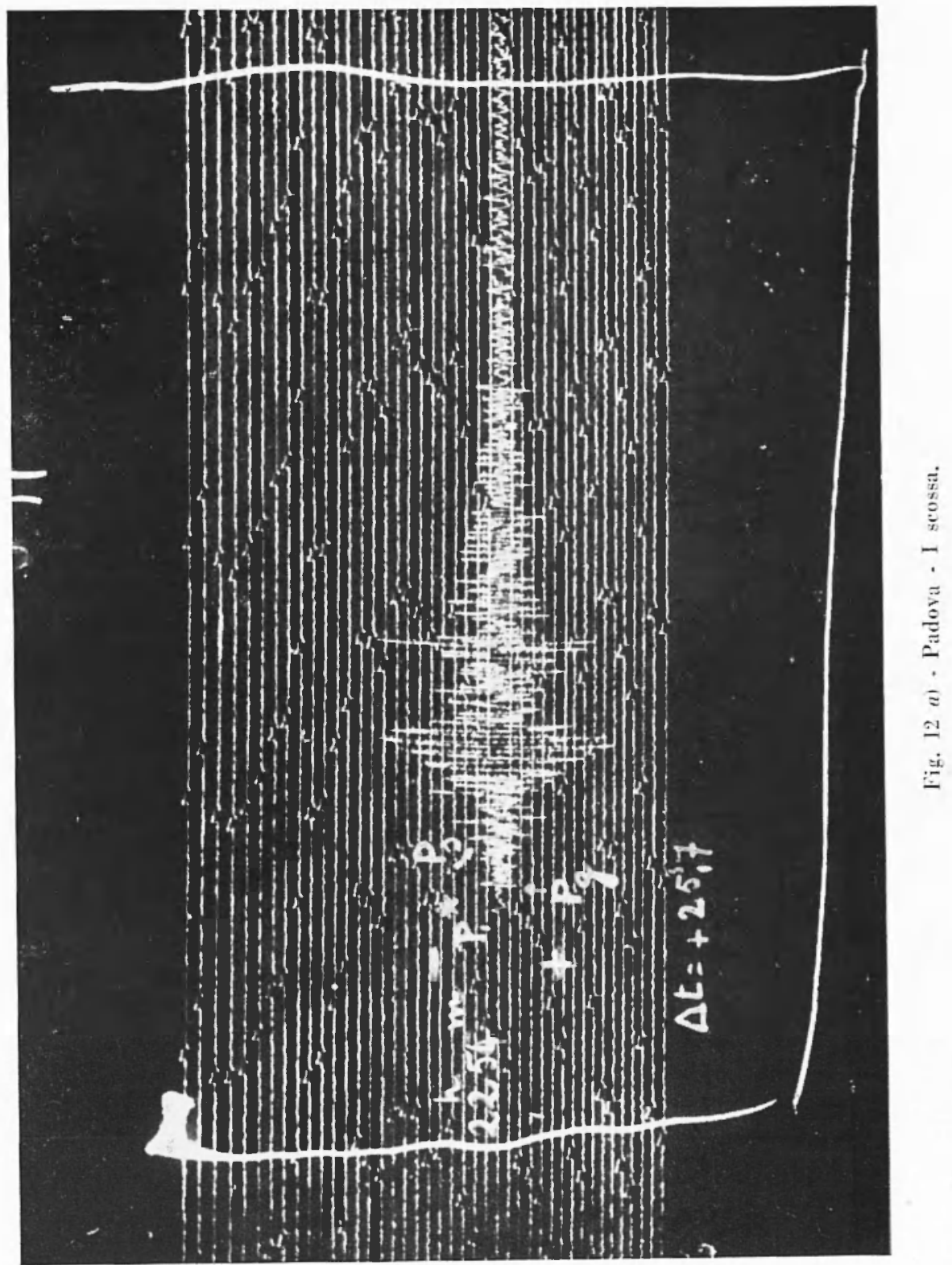




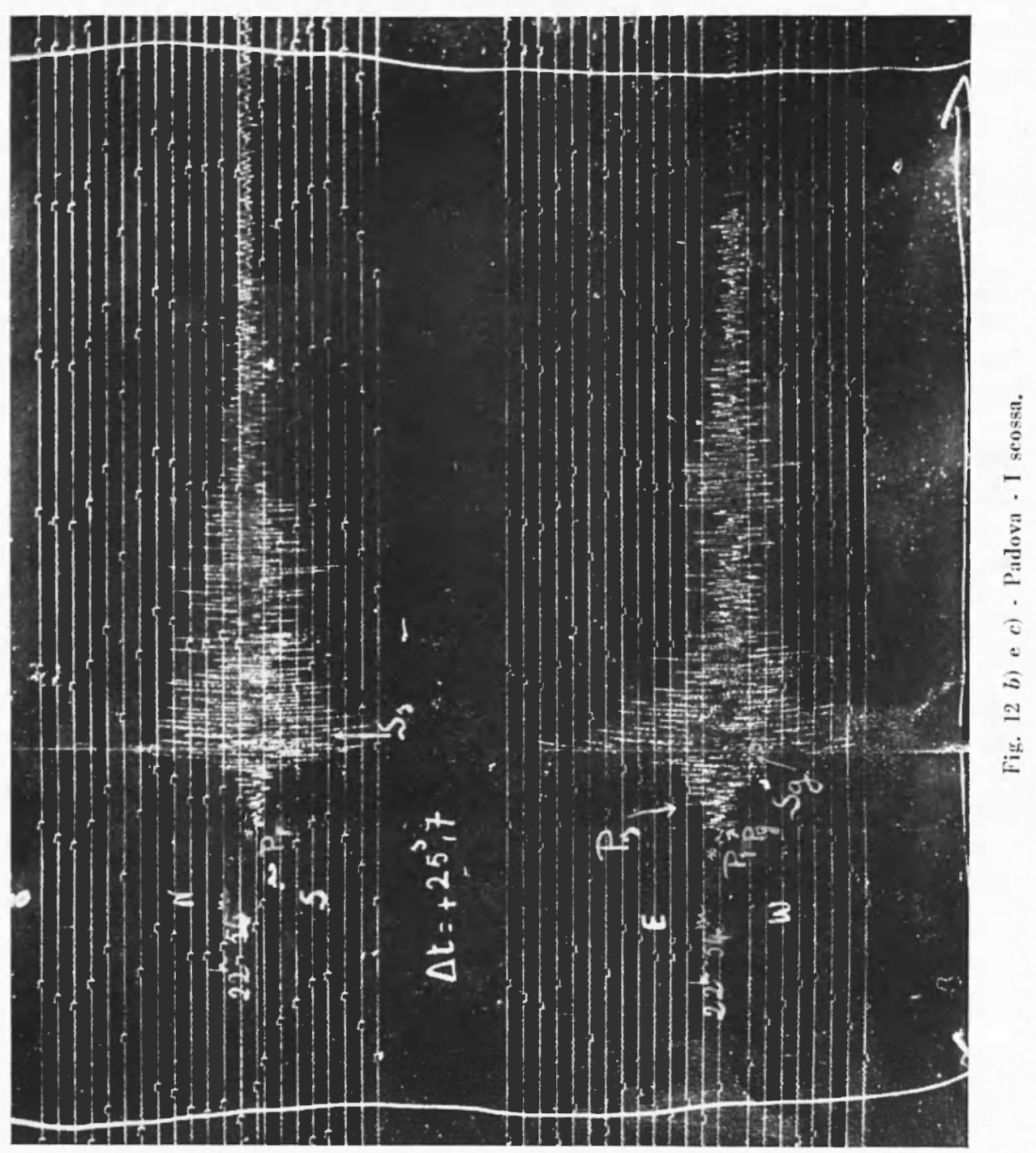


del terremoto deblo ricercarsi nella zona intermedia fra i sedimenti e lo strato del granito, fanno si che le oscillazioni sismiche interessanti gli strati di copertura siano di preponderante portata $\left({ }^{5}\right)$.

Specie nelle stazioni sismiche situate nella Val Padana o ai limiti della stessa, le oscillazioni interessanti lo strato sedimentario sono risultate le più ampie: cosi a Pavia, a Piacenza, a Bologna, a Padova, a Salò e a Trieste.

Seblene la cosa non potesse apparire in principio troppo agevole, ci siamo preoccupati di determinare le caratteristiche peculiari delle oscillazioni longitudinali e trasversali proprie degli strati sedimentari; e di vedere se dette oscillazioni interessavano pressoché esclusivamente la Val Padana o se venivano registrate a maggiore distanza.

Per quanto concerne la Val Padana, con le registrazioni ottenute a Salò, a Bologna e a Padova, abljiamo potuto determinare la velocità media di propagazione delle onde longitudinali relative ai sedimenti, onde che noi indichiamo con il simbolo $P_{\mathrm{s}}$. Tale velocità è risultata di $3,82 \mathrm{Km} / \mathrm{sec}$.

Qui cade opportuna un'osservazione. Questa velocità, come si è detto, ha senza dubljio il valore di una velocità media. È noto infatti che gli strati sedimentarii, costituenti il fondo della Val Padana, hanno caratteristiche che li distinguono — talvolta sensibilmente — gli uni dagli altri. Gli strati superficiali, risultanti da materiale sciolto, sono caratterizzati da deboli velocità di propagazione. Dette velocità tendono a crescere negli strati consolidati sottostanti.

Tale accrescimento però non è affatto lineare; non solo, ma si presenta fortemente saltuario da zona a zona. Consegue da ciò, sulla scorta della teoria della propagazione per "fronti guida", che la velocità media su indicata riguarda prevalentemente gli strati sedimentari più profondi, dove detta velocità presenta i massimi valori. Ciò è provato dal fatto che le vilorazioni interessanti i detti strati non si esauriscono in un impulso isolato, ma risultano costituite da tutta una serie di impulsi successivi, convogliati alle stazioni di osservazione dai "fronti guida " con velocità via via decrescenti.

Quanto si è detto, vale naturalmente anche per le corrispondenti onde trasversali, da noi indicate con il simbolo $S_{\mathrm{s}}$. Con i dati di Salo, Bologna, Padova, Pieve di Cadore e Trieste, abbiamo ottenuto per la velocità media delle onde $S_{\mathrm{s}}$ il valore di $2,38 \mathrm{Km} /$ sec.

Le onde $P_{\mathrm{s}}$ ed $S_{\mathrm{s}}$ hanno dato vistose, prolungate registrazioni in 
tutte le stazioni della Val Padana, nelle quali hanno costituito le fasi preponderanti.

Per le stazioni oltre la Val Padana la registrazione dei su detti tipi d'onda è continuata in maniera evidentissima. Solo è venuto a diminuire il numero degli impulsi, seguenti quelli iniziali, relativi alle stratificazioni di maggiore velocità. Ciò si spiega facilmente quando si osservi che la Val Parlana risulta dalla sovrapposizione di tutta una serie di sedimenti (fig. 5).

E chiaro che ai margini della Val Padana le stratificazioni più superficiali vengono via via a mancare, mentre si presentano come affioranti quelle più profonde.

Esempi chiarissimi di onde $P_{\mathrm{s}}$ ed $S_{\mathrm{s}}$ si sono ottenuti in tutte le stazioni europee: a Coira, a Neuchâtel, a Roma, a Stoccarda, a Jena, fino in Spagna. Anche le stazioni alpine hanno registrato chiari esempi di queste onde. $\dot{E}$ da ritenere pertanto che le stratificazioni caratterizzate da velocità medie di $4 \mathrm{~km}$ / sec per le onde longitudinali, e 2,5 $\mathrm{km}$ sec per le trasversali costituiscano una copertura generalmente diffusa, come del resto è provato dalle prospezioni sismiche esegnuite personalmente da mo di noi nelle Alpi Carniche e nel Cadore.

Il fatto che le oscillazioni interessanti gli strati sedimentarii abbiano assunto, per il terremoto in esame, tanta importanza può essere spiegato con le particolari modalità, con le quali il terremoto ha avuto origine. Infatti, come vedremo più avanti, lo scuotimento si è verificato con i caratteri di un brusco sollevamento secondo un asse quasi orizzontale e ai limiti fra i sedimenti e lo strato del "granito"; ciò ha fatto sì che gran parte dell'energia si sia propagata nelle stratificazioni superficiali guidata appunto dalla superficie limitante inferiormente le stratificazioni stesse.

VI1I. - Particolarmente lunga e laboriosa è risultata l'elaborazione delle onde che seguono le $P_{n}$ che si sogliono indicare con il simbolo $P^{*}$ (come le contrassegnava Conrad, da cui furono scoperte) o con $\boldsymbol{P}_{b}$, come alcuni le indicano al presente.

Tutti i tentativi intesi a esprimere i tempi di propagazione di queste onde con una sola dromocrona andarono falliti.

Era chiaro che non si trattava di un solo tipo d'onda. Esaminate le registrazioni con cura minuziosa, siamo riusciti a provare l'esistenza di due tipi d'onda interessanti due diverse stratificazioni profonde se- 
guenti quella del " granito ». In precedenza, nella zona europea, era stata indicata l'esistenza di una sola stratificazione seguente il " granito ") e limitata inferiormente dalla superficie di Mohorovicic. Non sappiamo se lo sdoppiamento da noi individuato ha carattere generale per tutto il Continente Europeo. Alcuni fatti lascerebbero ritenere che sia eflettivamente cosi. Non dobbiamo dimenticare che nelle numerose riccrche precedenti lo studio relativo all'onda $P^{* r}$ ha condotto sempre a risultati contrastanti e ha portato alla determinazione di velocità fortemente variabili, entro limiti che vanno da $6.29 \mathrm{Km}$ sec a $7,1 \mathrm{Km} /$ sec. Tale variazione potrebbe appunto essere spiegata con il fatto che non si trattava di uno strato unico: pertanto, il prevalere delle onde dell'uno o dell'altro strato portava ad inevitabili alterazioni della velocità.

Comunque nel nostro caso, è stato possibile individuarc due dromocrone.

Per la prima, riferita allonda che abbiamo indicato con $P^{m{ }_{1}}$, ci siamo serviti dei tempi ottenuti a Padova, Zurigo, Neuchâtel, Stoccarda, Strasburgo, Clermont-Ferrand, Parigi e Göttingen. L'equazione della relativa dromocrona è risultata:

$$
t=0.144065 \Delta+6,53 \text {. }
$$

Se ne deduce per le $P^{*}{ }_{1}$ una velocità di $6,94 \mathrm{Km}$ /sec. Calcolate le differenze fra i valori osservati e quelli calcolati, si è avuto per Padova $-0,2$ sec: Zurigo $-0,8$; Neuchâtel $+1,1$ : Stoccarda -0.3 ; Strasburgo -- 0,1; Clermont-Ferrand + 0,8; Parigi - 1,5; Göttingen $+1,0$.

La dromocrona delle $P_{2}^{*}$ è stata ottenuta con i tempi di registrazione avuti a Zurigo, Ravensburg, Neuchâtel, Stoccarda, Strasburgo, Jena, Parigi, Göttingen. La relativa equazione, riferita al tempo orinine $23^{\text {h }} 54^{\mathrm{m}} 24^{\mathrm{s}}, 4$, è la seguente:

$$
t=0,16415\lrcorner+4,20 \text {. }
$$

Ne consegue per le $P_{2}^{*}$ una velocità di $6,09 \mathrm{Km}$ sec. Calcolate le differenze fra valori osservati e calcolati, si è ottenuto per Zurigo - 1,1 sec; Ravensburg + 1.2; Neuchâtel $+1,2$; Stoccarda 0,0; Strasburgo $-1,4$; Jena $-0,8$; Parigi $-1,7$; Göttingen $+2,5$.

Gli scarti per la $P^{*}$ : sono leggermente superiori a quelli ottenuti per la $P^{*}{ }_{1}$.

Resta così provato che, almeno per una vasta zona intorno alla Val Padana, la stratificazione della crosta terrestre consiste di tre strati 
sovrapposti, caratterizzati da velocità per le onde longitudinali di 5,1 ; 6,1 e $6,9 \mathrm{Km} / \mathrm{sec}$ rispettivamente. Sopra lo strato del (granito », si trova una stratificazione di sedimenti, generalmente diffusa in tutta Europa : nei sedimenti più consolidati, le onde longitudinali presentano velocità medie dell'ordine di $3,8-4,0 \mathrm{Km} / \mathrm{sec}$.

Vedremo più avanti l'ordine di grandezza dello spessore di queste sedimentazioni in corrispondenza della Val Padana.

Ci siamo preoccupati di cercare pure le corrispondenti trasversali delle $P^{*}{ }_{1}$ e $P^{*}$.

Il risultato però è stato positivo solo per le $S^{*}{ }_{1}$. Dato il groviglio delle fasi, quale si presenta per le distanze considerate, un'individuazione delle $S_{2}^{*}$ è risultata pressoché impossibile.

Con i dati di Firenze, Zurigo, Ravensburg, Stoccarda, Strasburgo, Roma, Jena, Parigi e Gottingen, la dromocrona media delle $S^{*}{ }_{1}$ è risultata :

$$
I=\frac{\Delta}{4,2}+6,58
$$

IX. - Le onde $P_{n}$ hanno dato chiare registrazioni in parecchie stazioni sismiche. La relativa dromocrona fu determinata valendoci dei dati delle seguenti stazioni: Coira, Zurigo, Ravensburg, Neuchâtel, Stoccarda, Strasburgo, Roma, Clermont-Ferrand, Praga, Parigi, Messina, Kew.

L'equazione della dromocrona più probabile è risultata la seguente:

$$
t=(0,1226+0,0013)\lrcorner+9,65 \pm 0,74
$$

La velocità di propagazione (velocità apparente) delle onde $P_{11}$ risulta pertanto di $8,16 \mathrm{Km} / \mathrm{sec} \pm 0,09$.

Nella tabella II sono comprese le distanze epicentrali delle relative stazioni e la differenza fra i valori osservati e quelli calcolati, per le onde $P_{\mathrm{n}}$.

La velocità ottenuta per le $P_{n}$, come si vede, risulta conforme a quella cui si è pervenuti in precedenti ricerche.

$X$. - Anche le $S_{n}$ hanno dato chiari esempi di registrazione in parecchie stazioni sismiche. Per la determinazione della relativa dromocrona ci siamo serviti dei dati tratti dalle seguenti stazioni: Coira, Zurigo, Ravensburg, Neuchâtel, Stoccarda, Strashurgo, Praga, Parigi, Göttingen, Kew. 







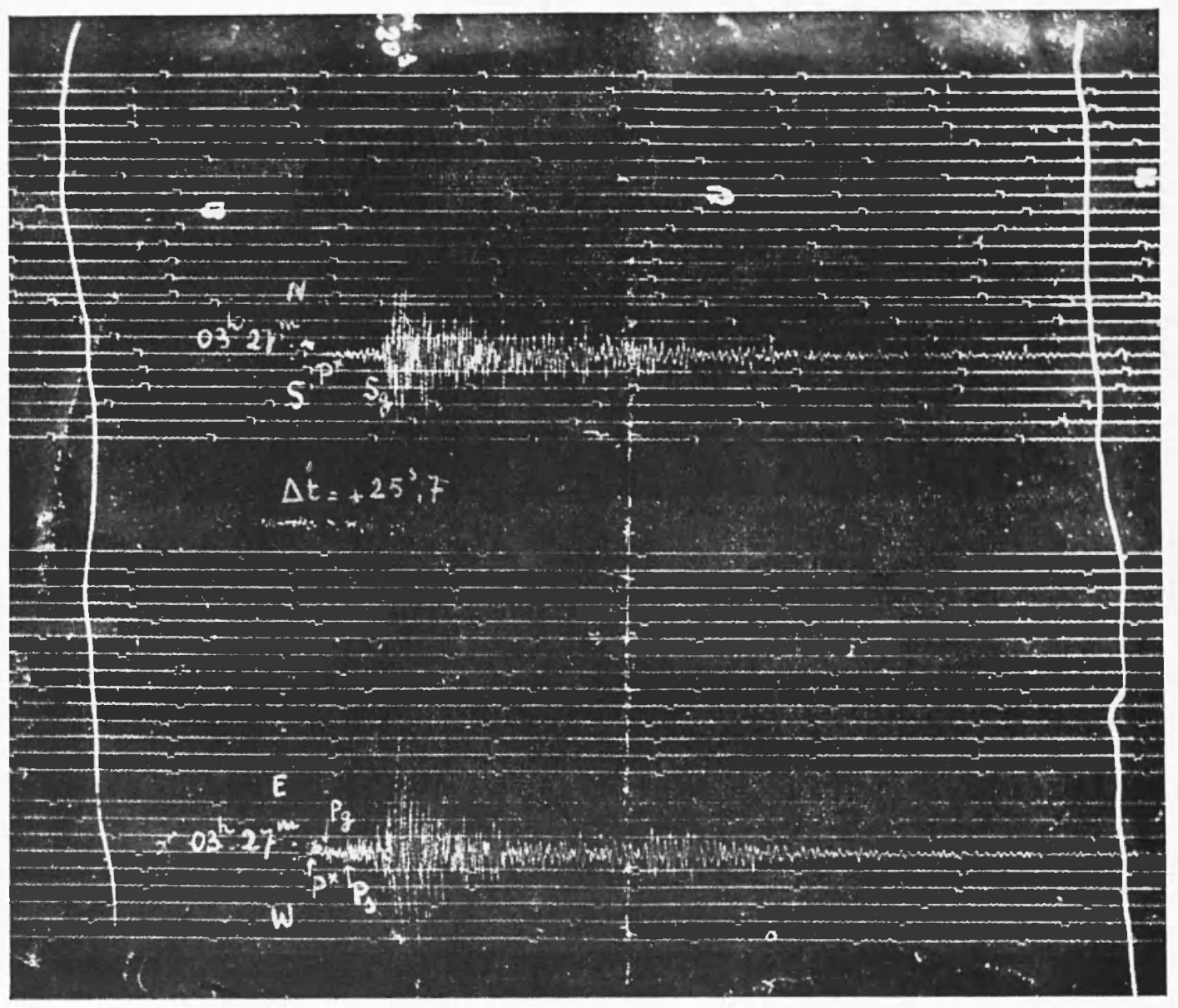

Fig. 13 b/ e c) - Padova - II scossal. 
Tabella II

\begin{tabular}{|l|c|c|}
\hline \multicolumn{1}{|c|}{ STAZIONI } & $\begin{array}{c}\Delta \\
\text { Distanze Epicen. }\end{array}$ & $\begin{array}{c}\mathrm{t}_{\mathrm{o}}-\mathrm{t}_{\mathbf{c}} \\
\mathrm{sec}\end{array}$ \\
\hline Coira & 171.7 & +0.80 \\
Zurigo & 242.5 & -0.48 \\
Ravensburg & 275.3 & +0.10 \\
Neuchâtel & 277.8 & -0.81 \\
Stoccarda & 378.3 & -0.13 \\
Strasburgo & 390.4 & -0.41 \\
Roma & +44.1 & -1.11 \\
Clermont-Ferrand & 508.5 & -1.19 \\
Praga & $6+0.2$ & +1.27 \\
Parigi & $66+.4$ & +2.30 \\
Messina & 930.2 & -1.96 \\
Kew & 1010.8 &
\end{tabular}

L'erfuazione della dromocrona piu probabile riferita all'ora all'ipocentro, è risultata:

$$
t=(0,2212+0,0013)\lrcorner+7,42 \pm 0,71
$$

Ciò porta ad una velocità per le $S_{\mathrm{n}}$ pari a $4,52 \mathrm{Km} / \mathrm{sec}$, dell'ordine ciò̀ di quella ottenuta in altri lavori.

Nella Tabella III a fianco di ogni stazione sono riportate le distanze epicentrali e le differenze fra i tempi osservati e quelli calcolati per le onde $S_{n}$.

TABELLA III

\begin{tabular}{|l|c|c|}
\hline \multicolumn{1}{|c|}{ STAZIONI } & $\Delta$ & $\begin{array}{c}\mathrm{t}_{\mathrm{o}}-\mathrm{tc}_{\mathrm{c}} \\
\mathrm{sec}\end{array}$ \\
\hline Coira & 171.7 & -0.30 \\
Zurigo & 242.5 & \pm 0.14 \\
Ravensburg & 275.3 & +0.71 \\
Neuchatel & 277.8 & +0.84 \\
Stoccarda & 378.3 & -0.91 \\
Strasburgo & 390.4 & -0.17 \\
Praga & 640.0 & -0.87 \\
Parigi & 664.4 & +1.43 \\
Gottingen & 694.8 & +1.88 \\
Kew & 1010.8 & +0.61
\end{tabular}

XI. - Profondità ipocentrale: Fra tutti gli elementi cbe caratterizzano un terremoto, quello di più difficile determinazione è la profondità dell'ipocentro. La precisione con cui è possibile determinare questa grandezza sovente non è molto spinta. Bastano infatti piccoli errori nella valutazione dei tempi di arrivo delle onde sismiche, per causare forti shalzi nel valore della profondità fornita dai calcoli. 
Le difficoltà poi crescono quando il terremoto si verifica in una zona eterogenea, ai margini di sistemi geologici di diversa natura. Il terremoto allo studio si presentava da questo punto di vista nelle condizioni più sfavorevoli. Esso si è verificato infatti al centro della Val Padana, in una zona caratterizzata da potenti stratificazioni alluvionali. Queste stratificazioni influenzano sensibilmente la propagazione delle onde sismiche in un raggio di alcune diecine di chilometri intorno all'epicentro. Facendo uso delle registrazioni delle stazioni sismiche poste entro detto raggio, non si può pertanto prescindere dall'influenza delle stratificazioni suddette.

Allo scopo di avere un primo risultato approssimativo che non risentisse dell'esistenza di questa coltre alluvionale, abliamo applicato un metodo che normalmente serve per la determinazione della profondità di terremoti di lontana origine. L'applicazione di questo metodo è stata fatta ai tempi delle onde $P$ ottenuti in stazioni sismiche funzionanti al di fuori della Val Padana. Il risultato ottenuto ha indicato una profondità nulla per il terremoto in parola. L'applicazione di un secondo metodo, più adatto per terremoti di vicina origine, ha portato alla stessa conclusione. Questo risultato, naturalmente, non va preso in senso assoluto: esso va interpretato nel senso di ritenere la scossa come originante nella zona limite fra la coltre alluvionale e la sottostante roccia compatta.

Con i metodi precedentemente accennati non era possibile ottenere dati più precisi. Comunque essi hanno servito a provare che il terremoto ha interessato essenzialmente la coltre superficialc.

Restava ora da escogitare un metodo che consentisse una valutazione più precisa della profondità.

In un primo momento abliamo fatto l'ipotesi - del resto attendibile - che la profondità ipocentrale coincidesse con il medio spessore della coltre alluvionale. In questa ipotesi si ottiene la seguente formula per il valore della profondita $h$ :

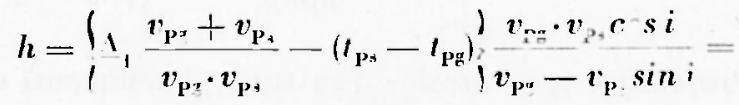

$$
\begin{aligned}
& =\frac{1}{\sqrt{\left(1 / v_{\mathrm{P} s}\right)^{2}-\left(1 / v_{\mathrm{Pg}}\right)^{2}}}\left\{\Delta_{1} \frac{v_{\mathrm{P} g}-v_{\mathrm{Ps}}}{v_{\mathrm{Pg}} \cdot v_{\mathrm{Ps}}}-\left(t_{\mathrm{P}_{\mathrm{t}}}-t_{\mathrm{Pg}}\right)\right\} \text {, }
\end{aligned}
$$

in cui $v_{\mathrm{P}_{\mathrm{g}}}$ e $v_{\mathrm{H}_{\mathrm{H}}}$ sono le velocità di propagazione delle onde longitudinali negli strati alluvionali e nel granito rispettivamente; $r_{\mathrm{p}}$ e $t_{\mathrm{g}}$ 
sono i corrispondenti tempi di registrazione alla distanza epicentrale $\dot{u}_{\text {: }}$.

Abbiamo applicato la formula alla stazione di Bologna, che ha fornito una delle registrazioni più chiare. Per un valore medio di $v_{\mathrm{p} *}$ $=3,5 \mathrm{Km} / \mathrm{sec}$, per $v_{\mathrm{p}:}=5,1 \mathrm{Km} / \mathrm{sec}$ e $\lrcorner_{1}=162,6 \mathrm{Km}$, poiché a Bologna risulta $t_{p_{\mathrm{s}}}-{ }^{\prime}{ }_{\mathrm{pg}}=13,6^{\mathrm{s}}$, si ottiene per $\boldsymbol{h}$ il valore di $5 \mathrm{Km}$ ca.

Un altro valore per la profondità ipocentrale poteva ottenersi ricorrendo alle differenze dei tempi di registrazione $S_{\mathrm{s}}-P_{\mathrm{s}}$, relativi alle onde trasversali e longitudinali proprie degli strati alluvionali, conosciuto il valore di $k$.

Tale valore è stato da noi determinato in 6,3. Questo però si riferisce a lunghi tragitti negli strati alluvionali e pertanto si deve ritenere come un valore medio massimo, in quanto interessa in prevalenza gli strati più profondi, che consentono maggiori velocità. Applicando a stazioni vicine all'epicentro - alle quali soltanto il metodo consente di ricorrere - detto valore di $k$ può portare a risultati approssimati per eccesso. Per l'applicazione abbiamo fatto ricorso ai dati delle stazioni di Pavia e Piacenza. Per Pavia si ha $S_{\mathrm{s}}-P_{\mathrm{s}}=6,2$. Fatto $k=6,3$, ne conseguirebbe per $h$ il valore di circa $12 \mathrm{Km}$, da ritenersi senz'altro eccessivo. Data la vicinanza di Pavia all'epicentro, è molto più probabile per $k$ un valore dell'ordine di 6 . Con tale valore per $k$, consegue per la profondità ipocentrale il valore di $5 \mathrm{Km}$ ca.

Piacenza dista dall'epicentro $\mathrm{Km}$ 44,8. Per essa la differenza $S_{\mathrm{s}}-P_{\mathrm{s}}$ è dall'ordine di 7,3 . Per $k=6,2$ consegue una profondità dell'ordine di $6,5 \mathrm{Km}$. Per un valore di $k$ minore, la profondita si annulla.

Possiamo concludere che la profondità ipocentrale può ritenersi di 3-5 Km ca.

XII. Stratificazione. - Il problema della stratificazione rivestiva un interesse speciale per la zona in cui il terremoto si ̀̀ verificato. Non era infatti possibile prescindere dalle stratificazioni superficiali alluvionali, che nella Val Padana raggiungono spessori notevoli. Bisognava quindi, prima di tutto, determinare un valore medio del complesso di dette stratificazioni. La qualifica di complesso è giustificata dal fatto che, in realtà - come è ben noto - le formazioni alluvionali consistono di un numero indeterminato di strati sovrapposti con caratteristiche cangianti da strato a strato. Sarebbe certamente arduo, se non impossibile, valutare la giacitura delle varie superficie separanti l'uno dall'altro strato, anche perché molto spesso non è il caso di parlare di vere e proprie superficie, ma bensi di zone di transizione, 


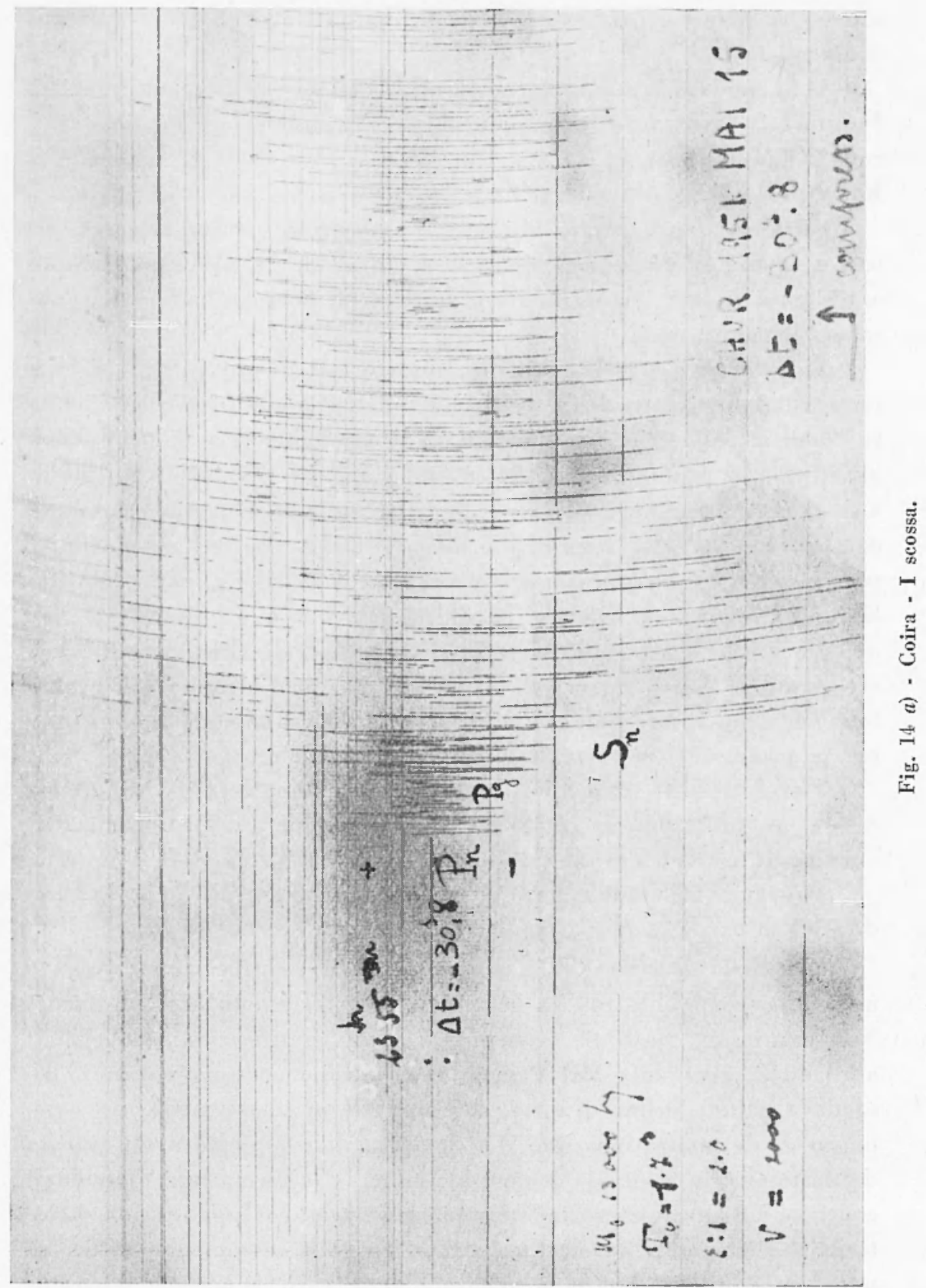


che non possono reagire come superficie quando sono sollecitate elasticamente. È quindi giocoforza, in uno studio di carattere generale, limi* tarsi alla ricerca di un valore approssimato dello spessore di tutto l'insieme delle stratificazioni alluvionali sovrapposte.

Ed è appunto ciò che abbiamo fatto.

Indichiamo con $d_{1}$ lo spessore medio delle stratificazioni alluvionali. Nella ipotesi $h=d_{1}$, abbiamo visto che l'ordine di grandezza dello spessore alluvionale è di $5 \mathrm{~km}$.

Con i dati di una stazione sismica, possiamo trarre un valore per $d_{1}$ conoscendo $\mathrm{i}$ tempi di registrazione delle onde longitudinali che hanno conservato il loro carattere dall'ipocentro alla stazione di osservazione e delle onde dello stesso tipo che si sono trasformate in trasversali in corrispondenza della discontinuità fra il granito e l'alluvionale.

Se indichiamo con $\delta_{1}$ la differenza fra $i$ tempi di registrazione della seconda e della prima onda in parola, si ottiene:

$$
\delta_{1}=d_{1} / v_{\mathrm{Pg}}\left(\sqrt{\left(v_{\mathrm{P}_{\mathrm{g}}} / v_{\mathrm{Ss}}\right)^{2}-1}-\sqrt{\left(v_{\mathrm{Pg}} / v_{\mathrm{P}^{*}}\right)^{2}-1}\right)
$$

dove $\mathrm{i}$ simboli hanno significato manifesto. Fatta l'applicazione alla stazione di Bologna, dove risulta ${ }_{1}=1^{\mathrm{s}, 2}$, si ottiene

$$
d_{1}=6 \mathrm{~km} \text {. }
$$

Lo strato alluvionale avrebbe quindi nei pressi di Bologna, nella direzione Bologna-epicentro, uno spessōe complessivo dell'ordine di $6 \mathrm{~km}$.

Questo risultato viene a dare una conferma all'ipotesi - di cui ci siamo serviti in precedenza - che il movimento sismico si sia verificato verso la base delle stratificazioni alluvionali.

Ottenuto un valore medio approssimativo per il complesso delle stratificazioni superficiali, ci siamo preccupati di passare alla determinazione delio spessore medio del sottostante strato del "granito ».

Fra i varii metodi che potevano servire allo scopo, nessumo dava affidamento di condurre ad un risultato attendibile, attesa la speciale situazione geologica che ha caratterizzato il terremoto in esame. $\mathrm{Ab}$ biamo preferito, anche questa volta, fare ricorso ai dati di una stazione (Bologna) posta ai margini della Val Padana, prescindendo dalla sua distanza epicentrale.

Indichiamo con $d_{1}, d_{2}$ gli spessori degli strati alluvionali e del (granito ). Consideriamo il tempo di registrazione dell'onda $P_{1}$, che 


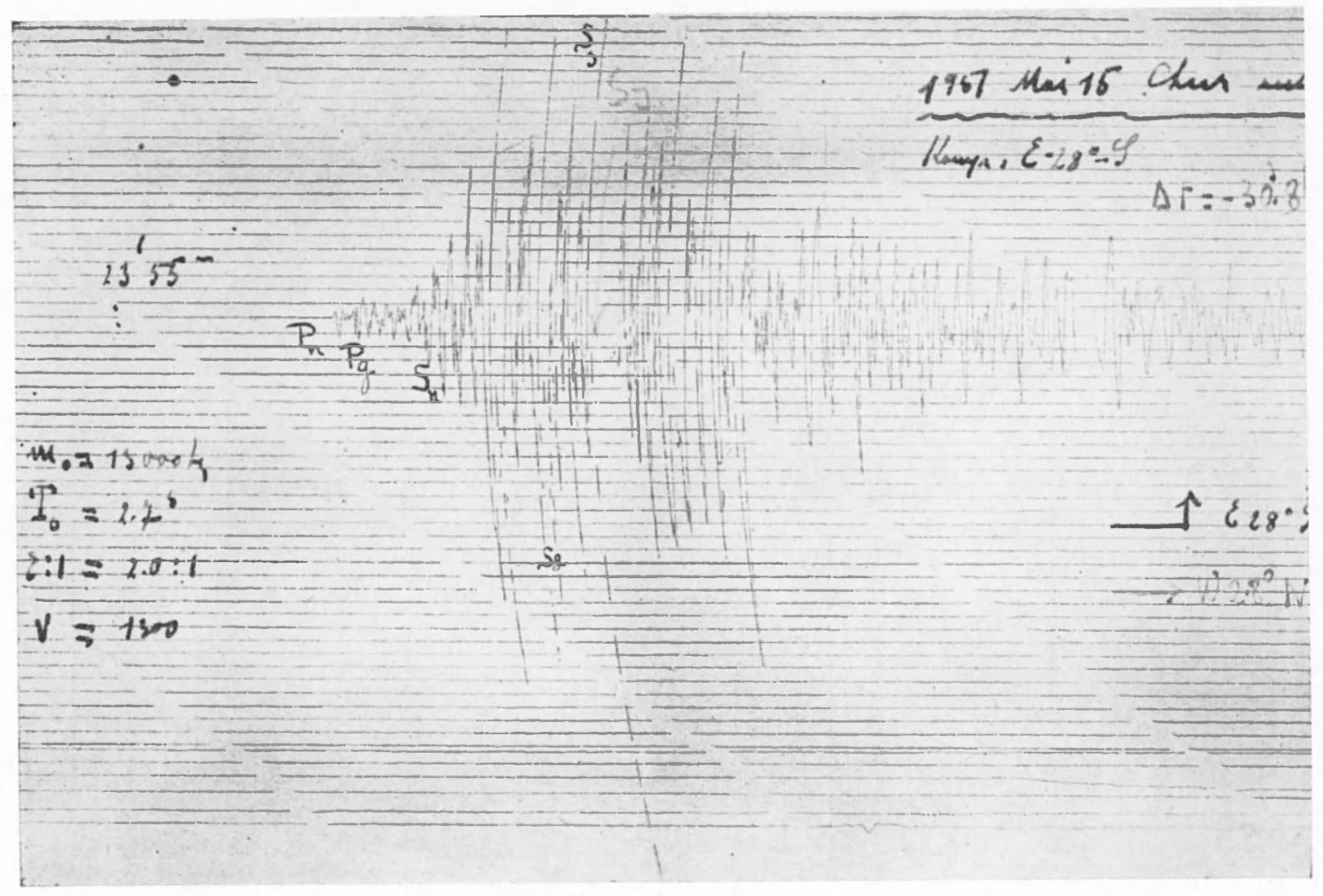

Fig. 11 b) - Coira - 1 scossa. 
interessa lo strato sopportante il "granito " e l'analogo tempo di registrazione dell'onda dello stesso tipo che da longitudinale si mula in trasversale passando nello strato del "granito». Si ottiene allora:

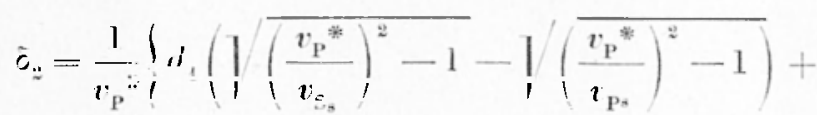

$$
\begin{aligned}
& \left.+d_{2}\left(\sqrt{\left(\frac{v_{\mathrm{P}}^{*}}{v_{\mathrm{S} z}}\right)^{0}-1}-\sqrt{\left(\frac{v_{\mathrm{P}}{ }^{*}}{v_{\mathrm{P} g}}\right)^{2}-1}\right)\right\}
\end{aligned}
$$

dove $\delta_{2}$ rappresenta la differenza fra $\mathrm{i}$ tempi di registrazione del secondo e del primo tipo d'onda sopra considerati. Gli altri simboli hammo significato ovvio.

Abbiamo fatto l'applicazione della [7] ( ${ }^{1}$ ) alla stazione di Bologna, per la quale risulta $\hat{c}_{2}=2$ s, 5 . Allora per $v *=6,1 \mathrm{~km} / \mathrm{sec}, v_{p \mu}=5,1$ $\mathrm{km} / \mathrm{sec}, v_{\mathrm{sg}}=3,1 \mathrm{~km} / \mathrm{sec} i_{\mathrm{ps}}=3,8 \mathrm{~km} / \mathrm{sec}, v_{\mathrm{s},}=2,4 \mathrm{~km} / \mathrm{sec}, d_{1}=$ $6 \mathrm{~km}$ consegue

$$
d_{\underline{2}}=8,5 \mathrm{~km} \text {. }
$$

Lo spessore dello strato del "granito" risulterebbe quindi dell'ordine di 8-9 km, almeno nella direzione Bologna-epicentro; valore quindi nettamente inferiore a quello che si osserva nelle limitrofe regioni alpina e appenninica. Ció lascia presumere che i susseguenti strati basaltici debhano avere uno spessore complessivo magrgiore di quello che, precedenti ricerche, hanno fornito per gli analoghi strati sottostanti le Alpi e l'Appennino.

XIII. Distribuzione superficiale dei movimenti iniziali delle onde longitudinali. - ì noto che dal verso di spostamento in superficie delle onde longitudinali, quando il numero delle stazioni sismiche intorno

(1) Indicando con $i$ l'ordine di successione dello strato e con $\mathrm{V}_{\mathrm{i}}, v_{\mathrm{i}} \quad i_{i}=1,2, \ldots$ n) le relative velocità delle onde longitudinali e trasversali rispettivamente, avremo in generale, se $d_{i} \dot{e}$ lo spessore dello strato considerato:

$$
\begin{aligned}
& \delta_{\mathrm{n}}=1 / V_{\mathrm{n}+1}\left\{d _ { 1 } \left(V / \overline{\left(\frac{\mathrm{n}+1}{v_{1}}\right)^{2}-1}-V \overline{\left(\frac{\left.V_{\mathrm{n}+1}\right)_{1}^{2}-1}{l_{1}}\right)}+d_{2}\left(\sqrt{\left(\frac{V_{\mathrm{n}+1}}{r_{2}}\right)^{2}-1}-\right.\right.\right. \\
& \left.\left.-\sqrt{\left(\frac{V_{\mathrm{n}+1}}{V_{2}}\right)^{2}-1}\right)+\ldots \ldots+d_{\mathrm{n}}\left(\sqrt{\left(\frac{V_{\mathrm{n}+1}}{r_{\mathrm{n}}}\right)^{2}-1}-\sqrt{\left(\frac{V_{\mathrm{n}+1}}{V_{\mathrm{n}}}\right)^{2}-1}\right)\right\} .
\end{aligned}
$$

Qualora le onde relative fossero facilmente individuabili, la [8] potrebbe servire a determinare lo spessore medio delle stratificazioni che succedono al "granito". 

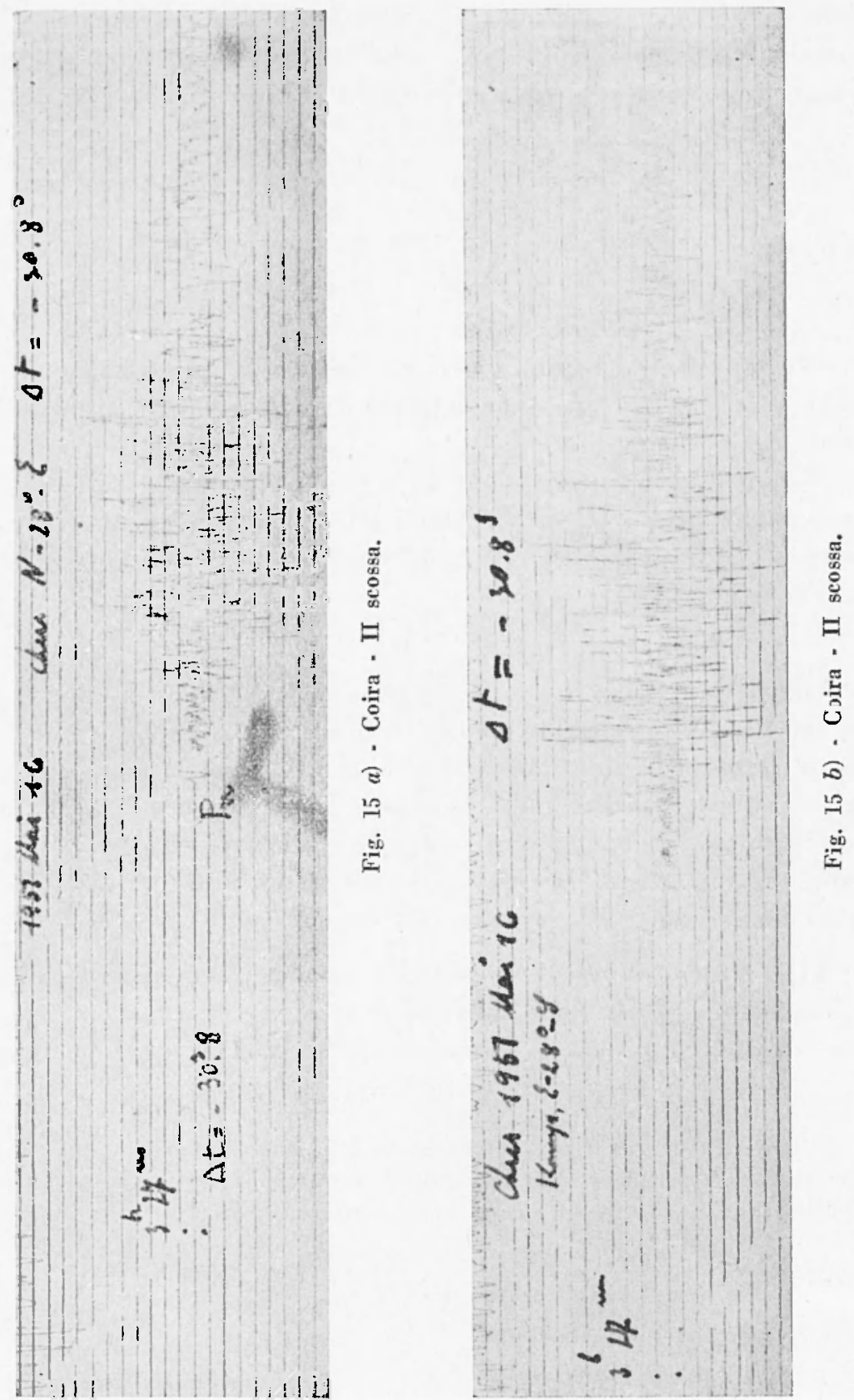


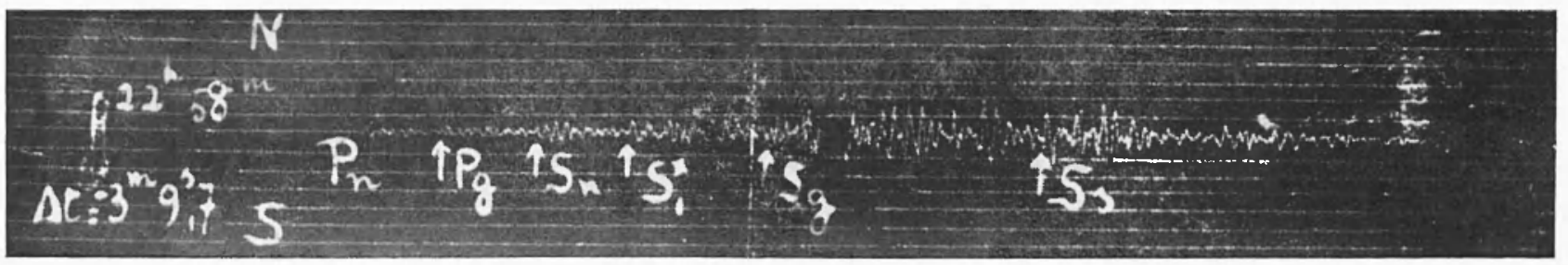

Fig. 16 (i) . Pieve di Cidore - I scossa.

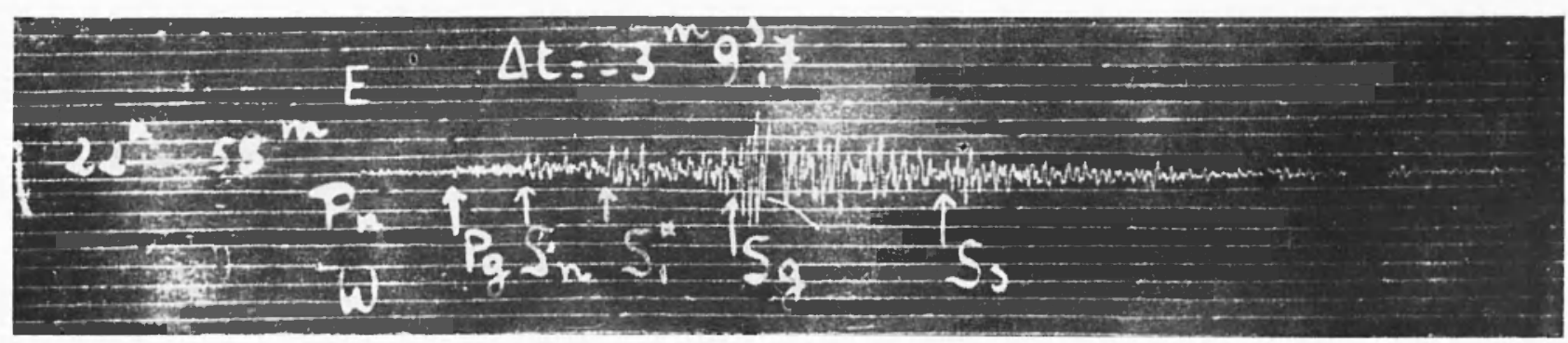

Fig. $16(b)$. Pieve di Cadore - I scossa.

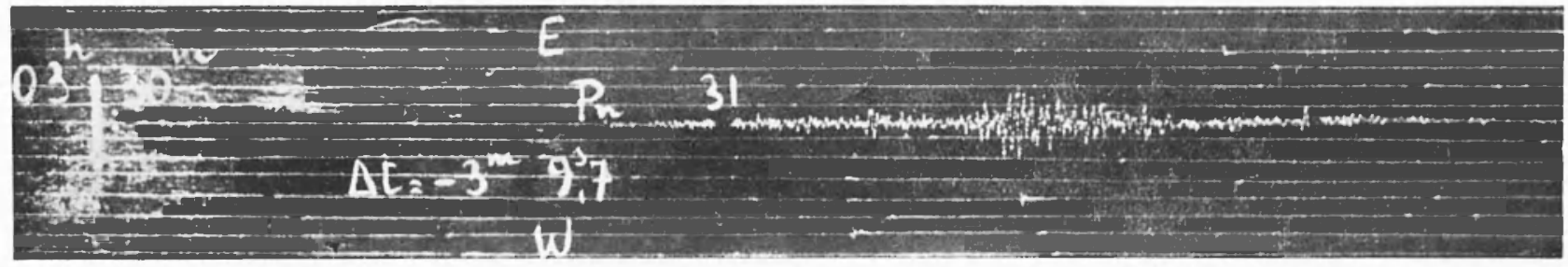

Fig. 16 cl. Pieve di Cadore. II scossa. 
all'epicentro è sufficientemente grande, è possibile risalire alla natura fisica della scossa all'ipocentro; stabilire, in altri termini, se la scossa è dovuta a frattura, a sprofondamenti di strati, a sollevamento o è di carattere esplosivo.

La posizione dell'epicentro del terremoto in esame era tale da consentire un'indagine del genere, data la densità delle stazioni sismiche nell'Italia settentrionale e nel centro Europa. La figura 6 riassume il risultato di questa indagine. In Europa, non è mai stata osservata una distribuzione superficiale dei movimenti iniziali come quella provocata dal terremoto del 1951 in Val Padana. I terremoti appenninici, nella loro quasi totalità, danno distribuzioni superficiali caratterizzate da una prima zona pressoché circolare intorno all'epicentro comprendente compressioni. II che si giustifica con bruschi movimenti di sollevamento, quali vengono confermati da ricerche geologiche.

La zona che ci interessa però, almeno nei tempi storici, è stata sempre considerata asismica; e comunque non ci risulta che, geologicamente, sia da considerarsi in fase di sollevamento. Resta ad ogni modo il fatto che il terremoto in esame è stato provocato da una rottura di equilibrio che ha determinato in una area, notevolmente sviluppata nella direzione Nord-Ovest, un movimento di compressione. L'area stessa si presenta nettamente decentrata rispetto all'epicentro, il che lascia presumere che la causa perturbante abbia agito - con una brusca pressione - secondo un asse fortemente inclinato rispetto alla verticale e in direzione Sud Est-Nord Ovest. A questo riguardo è interessante il confronto con le osservazioni macrosismiche.

Premettiamo che l'elaborazione di dette osservazioni è stata fatta da uno di noi all'insaputa del risultato ottenuto nell'indagine del senso di movimento in superficie delle onde longitudinali.

E noto che le stratificazioni alluvionali di una certa potenza esaltano le perturbazioni sismiche. Avviene così che, generalmente, gli abitanti di estese pianure alluvionali avvertono sensibilmente scotimenti che, altrove, passano pressoché inosservati.

Terremoti nella Val Padana p. es. (o ai margini di essa) risentono sempre di questa caratteristica e le relative isosiste risultano allungate nel senso dell'asse della valle stessa.

Nel terremoto in questione invece, il meccanismo secondo cui la scossa si è verificata ha prevalso sulla maggiore propagabilità dell'energia negli strati superficiali della Val Padana, così da imporre isosiste 
allungate nella direzione NW-SE, conformemente alla distribuzione superficiale del movimento iniziale delle onde longitudinali.

Quanto si è detto per la prima scossa vale, con qualche modifica, anche per la seconda scossa verificatasi verso le ore 3 e 30 del mattino successivo. La fig. 2 ne dà le caratteristiche dal punto di vista macrosismico.

Per ciò che concerne $\mathrm{i}$ movimenti iniziali in superficie, attesa la minore intensità della scossa, una delimitazione sulla base dei dati delle stazioni lontane, non è stata possibile. Le registrazioni ottenute dalle stazioni centro-europee, ci hanno però consentito di tracciare, con sufficiente esattezza, la linea che limita le compressioni dalle dilatazioni. Come risulta dalla fig. 7 , tale delimitazione, nella zona epicentrale, è leggrermente diversa da quella ottenuta per la prima scossa: a Pavia, p. es., anziché una compressione come per la prima scossa, si è avuta una nettissima dilatazione.

Comunque il meccanismo secondo cui la scossa si è verificata, ha strette analogie con quello delle scosse precedenti; soltanto l'asse delle zone interessate dalle compressioni ha avuto, rispetto al Nord, una inclinazione leggermente diversa.

XIV. Sulla sismicità del Lodigiano e sulle probabili cause delle scosse verificatesi nella notte fra il 15 e il 16 maggio 1951. - La scossa avvenuta il 15 maggio 1951 alle $23^{\text {li }}$ ca. e la replica delle $2^{\text {1 }} 30^{\mathrm{s}} \mathrm{ca}$. del mattino successivo hanno avuto il loro epicentro a SE di Lodi.

$\mathrm{Ci}$ siamo preoccupati di ricercare notizie sulla cronologia sismica della zona.

Solo il Baratta, nella sua voluminosa opera sui terremoti in Italia $\left({ }^{6}\right)$ riporta una notizia circa un terremoto rovinoso che sarebbe avvenuto in Lodi nell'anno 290 dell'èra volgare. Il Baratta però esprime qualche dubbio sulla veridicità di questa notizia, anche perché successivamente non è più stato segnalato nessun movimento sismico con origine nel Lodigiano; tanto è vero che il citato autore nella cartina sulla sismicità dell'Italia settentrionale — cartina della quale la figura 8 riporta il tratto che a noi interessa - Lodi e il Lodigiano non figurano in nessuna area sismica, nemmeno in quelle secondarie a centro non determinato. Mario Baratta riprese l'argomento sulla sismicità della Penisola negli ultimi anni della sua vita $\left({ }^{7}\right)$ e, nel 1936, pubblicò per conto dell'Accademia Nazionale dei Lincei (Commissione Italiana per lo Studio delle grandi Calamità) un nuovo lavoro su " I terremoti in 




Fig. 17 b) - Zurigo - I scossa. 
Italia ", lavoro corredato da una nuova carta sismica d'Italia. Anche in questa carta, di cui la figura 9 riporta il tratto che a noi interessa, Lodi e il Lodigiano vengrono esclusi da ogni area sismica.

A quale causa dunque va attribuito il terremoto del 15 maggio 1951, che ha avuto caratteristiche superficiali tanto diverse da quelle che caratterizzano i terremoti del confinante sistema alpino? ì noto che ben difficilmente si formano sulla terra nuove aree sismiche, in conformità con il detto: "Dove ha tremato tremerà ). Non è quindi del tutto azzardato ricercare l'origine di questa scossa in cause accidentali. Osserviamo che l'epicentro cade precisamente nei pressi di Caviaga (fig. 10). In questa località esistono pozzi metaniferi, da cui si estraggono giornalmente notevoli quantità di gas metano che vanno dai $10.000 \mathrm{~m}^{3}$ ai $300.000 \mathrm{~m}^{3}$. La profondità degli strati metaniferi in questa zona è di 1250-1450 m ca. dalla superficie e la pressione massima alla bocca dei pozzi varia dai 130 ai $140 \mathrm{~kg} / \mathrm{cm}^{2}$. Esistono però nel Lodigiano altri campi metaniferi, dove si verificano analoghe estrazioni di metano, con pressioni alla bocca dei pozzi dell'ordine di quelle sopra riportate, se non superiori. Tale estrazione dura da anni ormai: la decompressione in atto nella zona attiva è quindi notevole. Ci si può fare un'idea di tali decompressioni, quando si pensi che l'estrazione di un milione di metri cubi di gas al giorno, ad una pressione di $100 \mathrm{~kg} / \mathrm{cm}^{2}$, comporterebbe un lavoro giornaliero di $10^{20} \mathrm{erg}$. Decompressione veramente cospicua quindi.

Si tratta naturalmente di uno sprigionamento di un'energia graduale e non istantanea, come avviene nei terremoti. Non si può negare comunque che, nel sottosuolo, abbia una funzione di equilibrio anche questa enorme quantità di gas compresso.

Tale funzione varierà a seconda della struttura geologica della regione, la quale potrebbe essere tale da annullare praticamente ogni effetto perturbante, come potrebbe facilitare la tendenza verso nuove posizioni di equilibrio, le quali non sempre vengono raggiunte gradualmente.

Che quanto è stato sopra detto abbia una base di attendibilità può essere provato anche per altra via. Nel marzo del 1949 nelle vicinanze della frazione di Basiasco (Lodigiano), durante le normali operazioni di trivellazione per la ricerca del metano, una improvvisa e violenta eruzione di gas si sprigionò da uno dei pozzi di sondaggio. L'eruzione del gas, il quale si innalzava dalla bocca del pozzo con una pressione di circa 120 atm., poté essere domata solo dopo molti giorni 


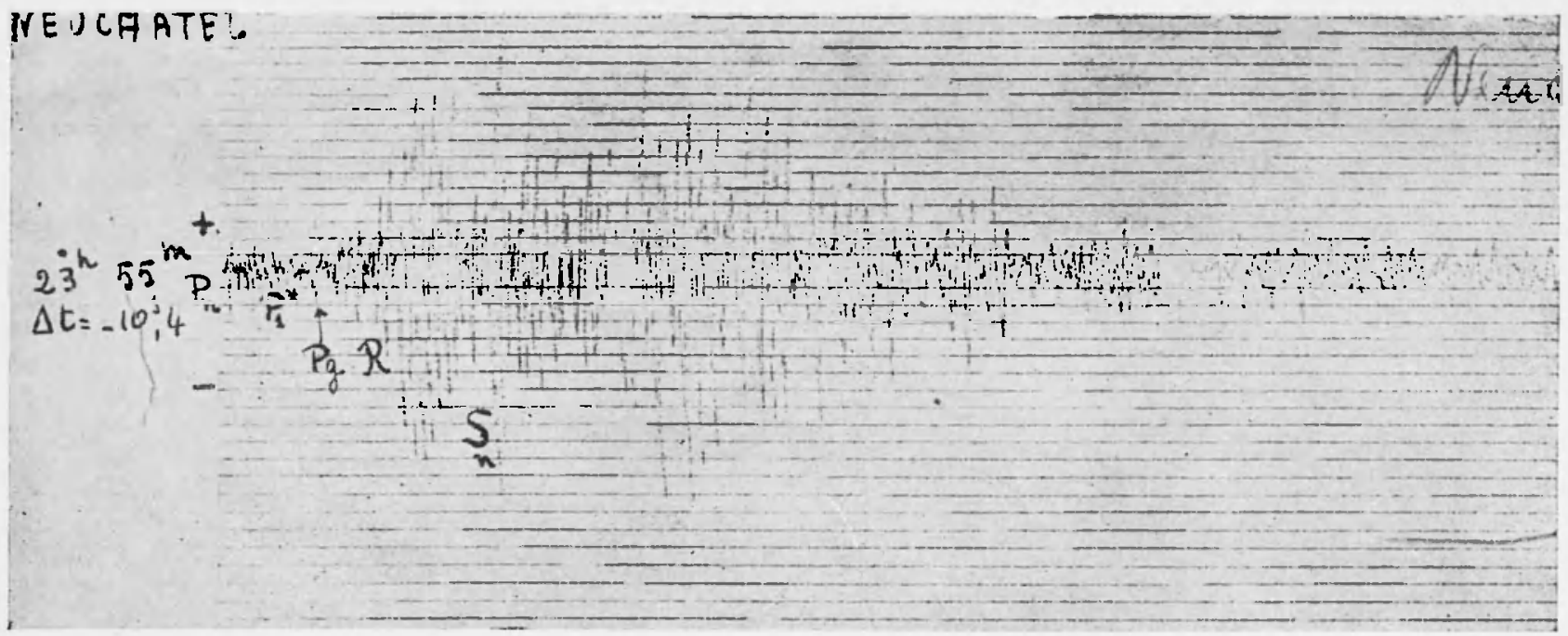

Fig. 18 a) - Neuchâtel - I scossa.

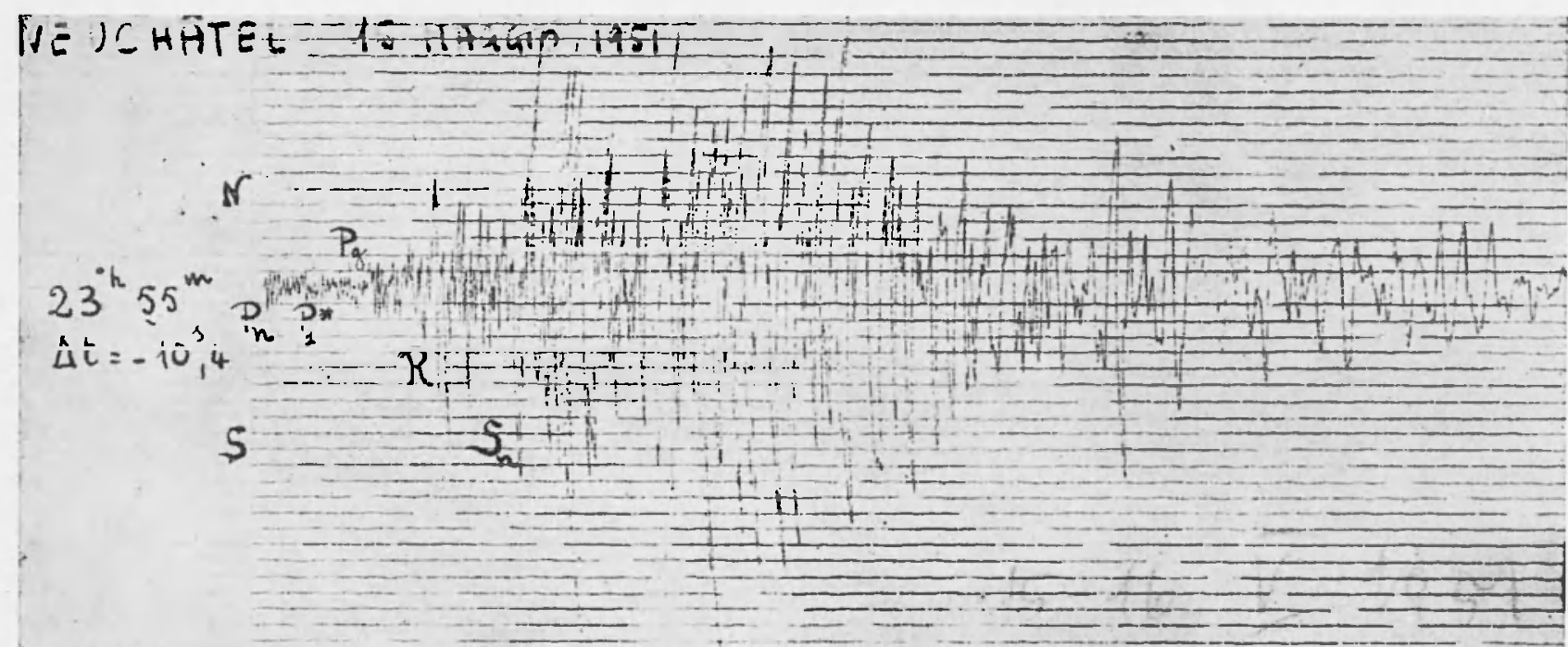

Fig. 18 b) - Neuchâtel - I scossa.

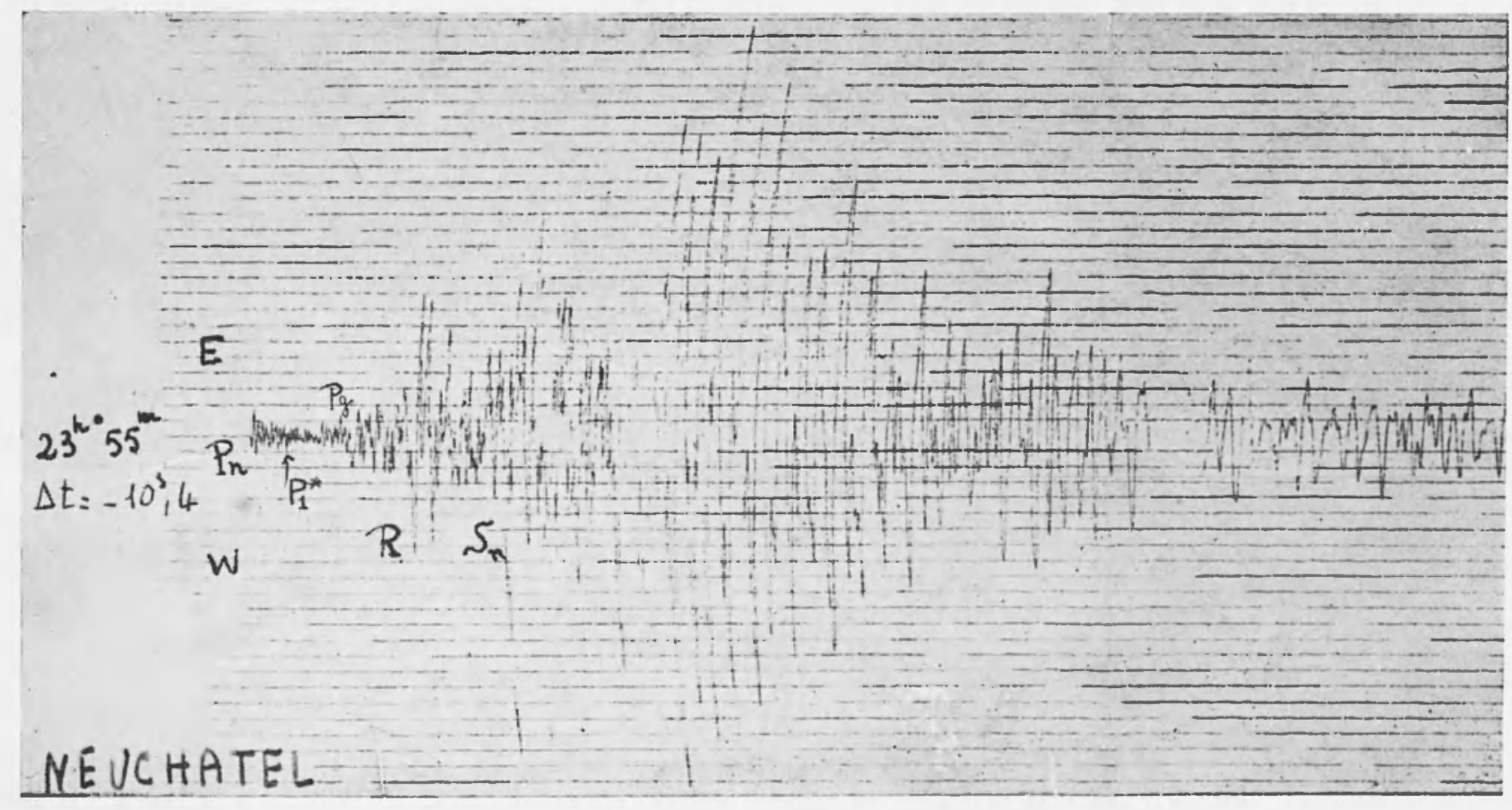

Fig. 18 c) - Neuchâtel - I scossa. 

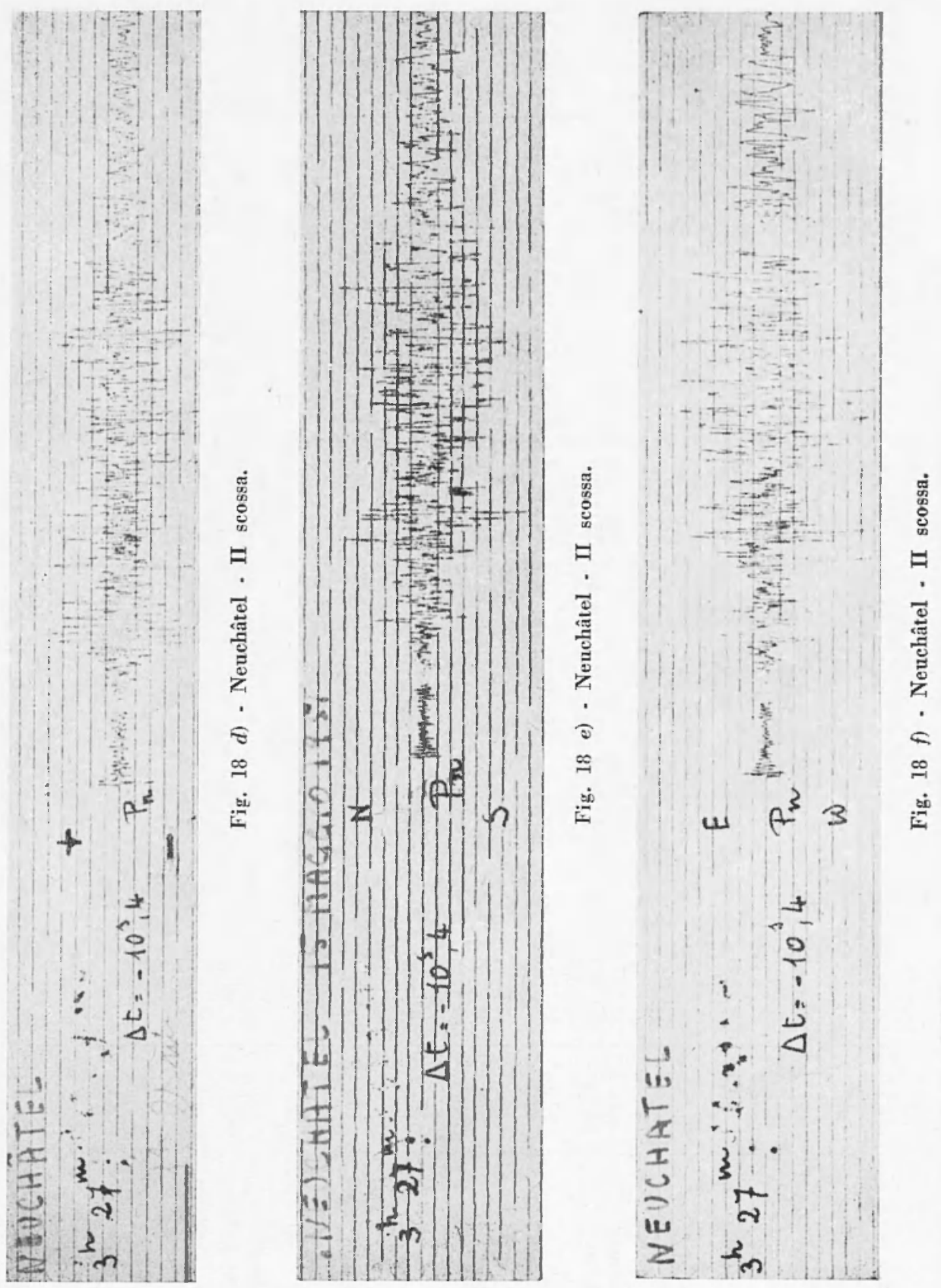


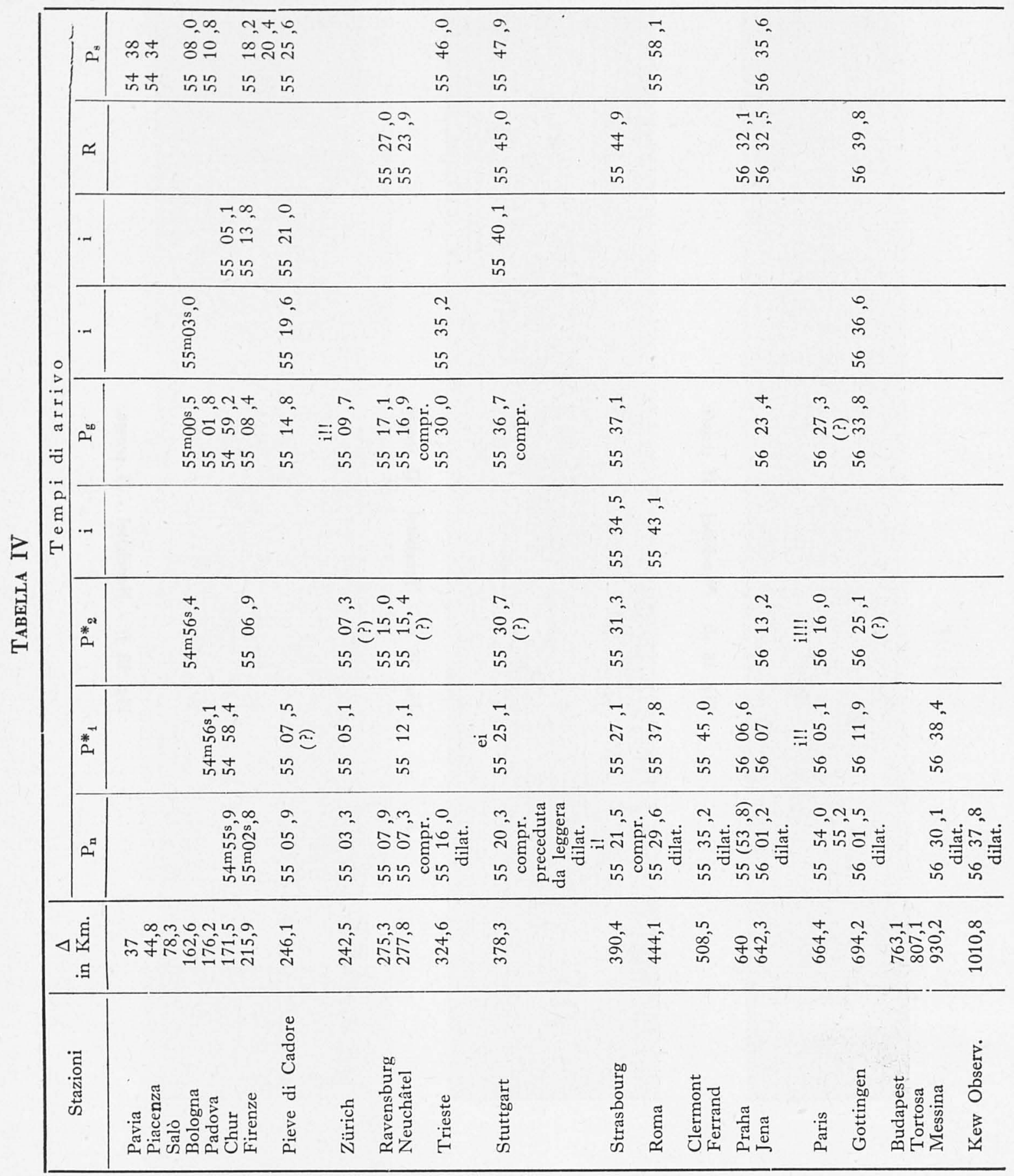



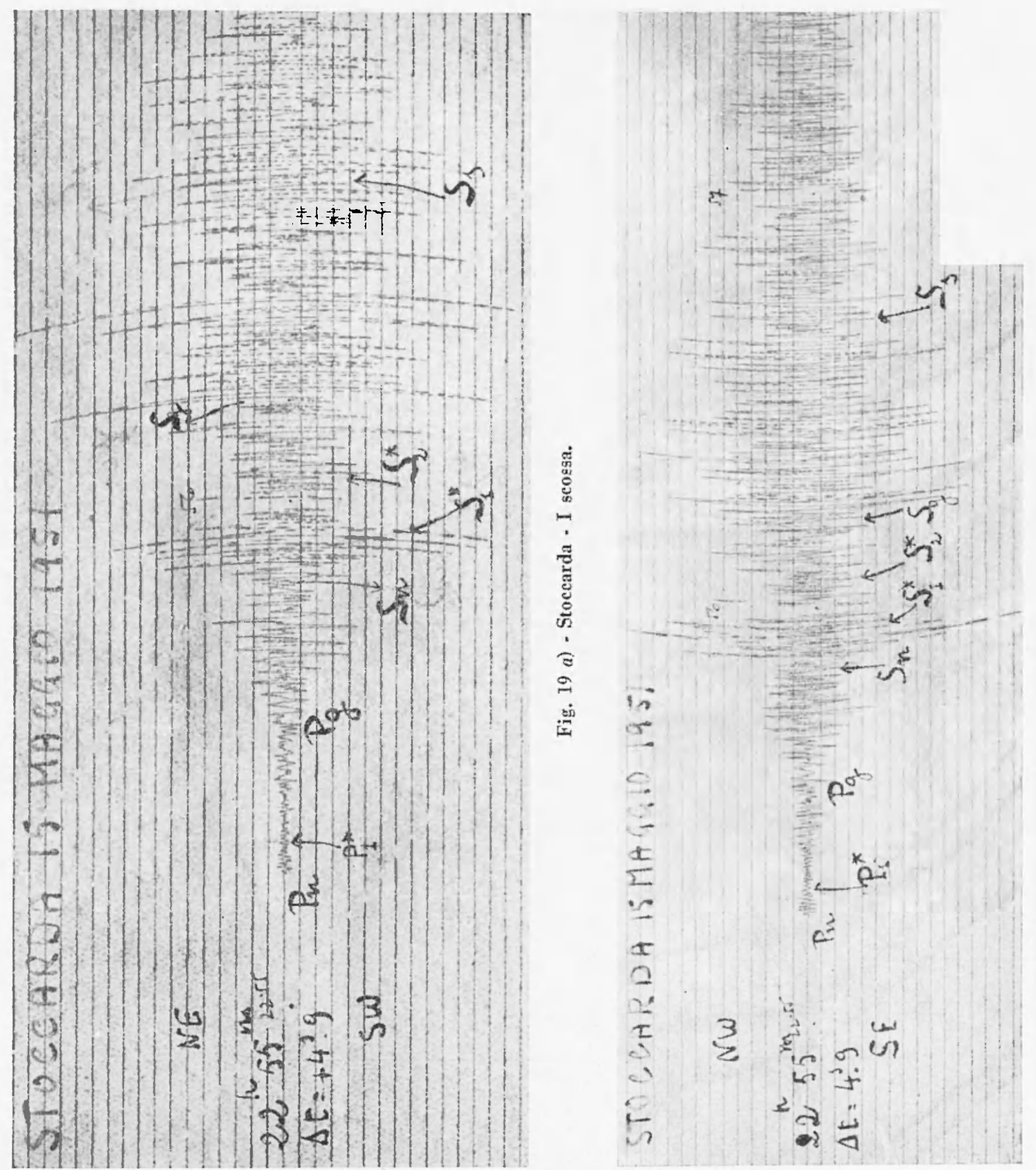

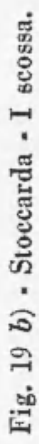


Tabella IV (seguito)

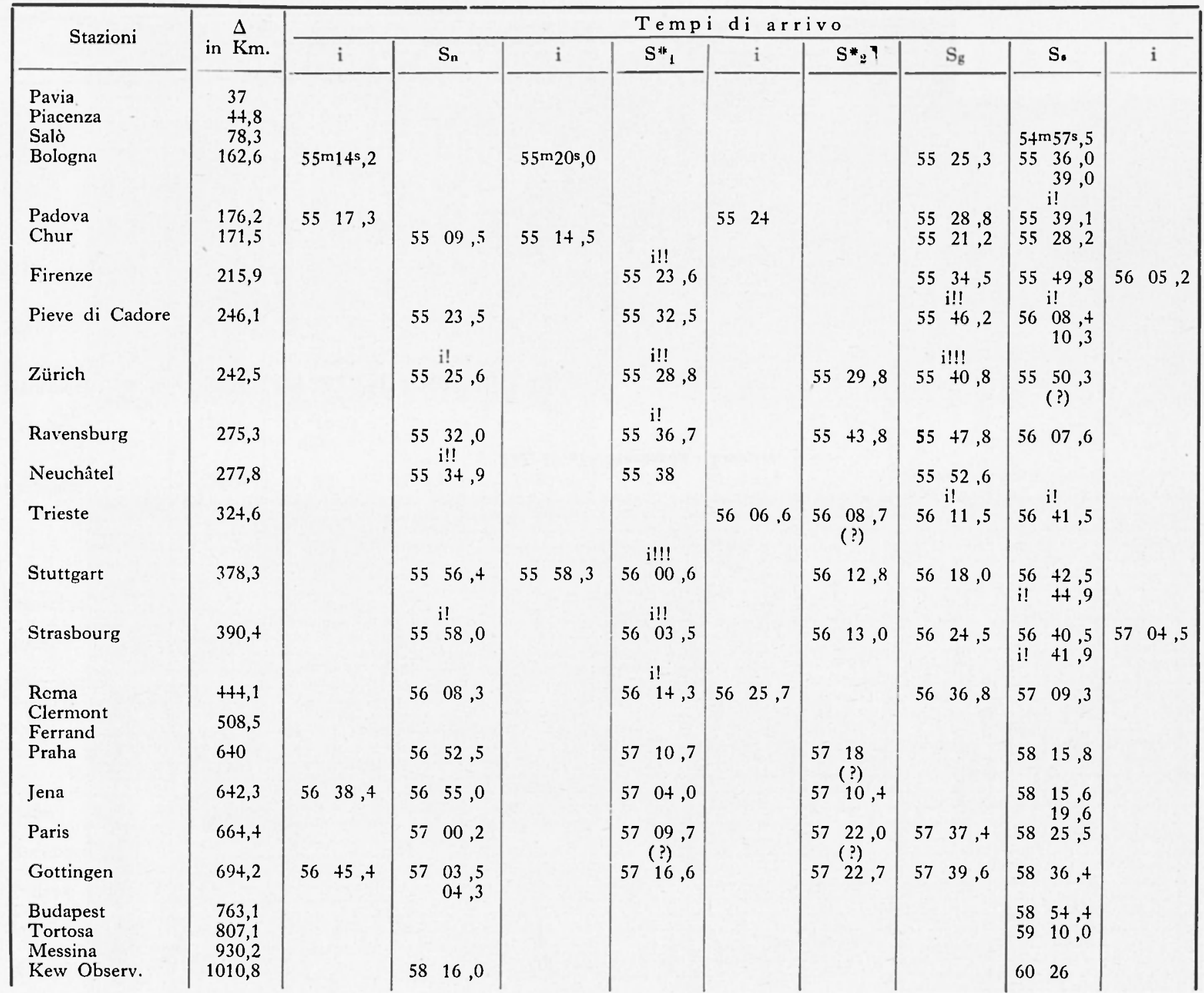




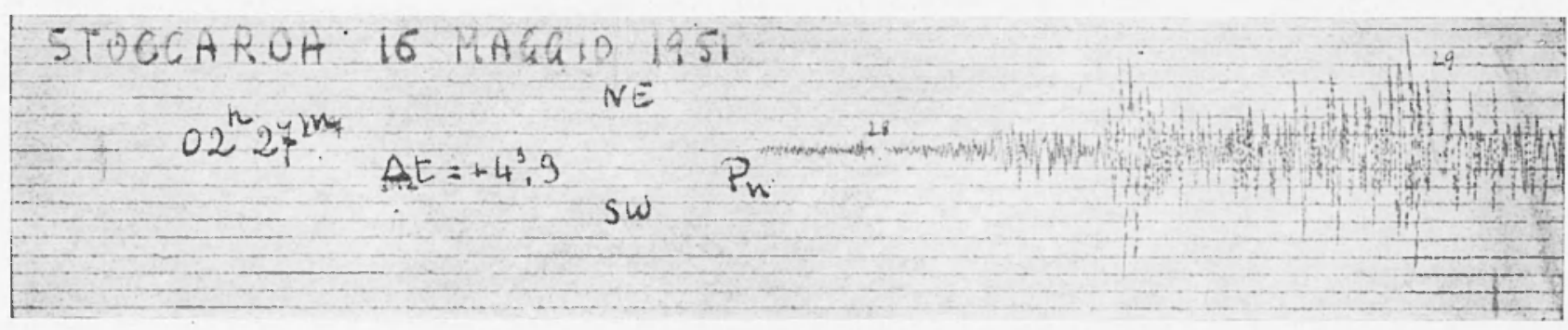

Fig. 19 c) - Stocearda - II scossa.

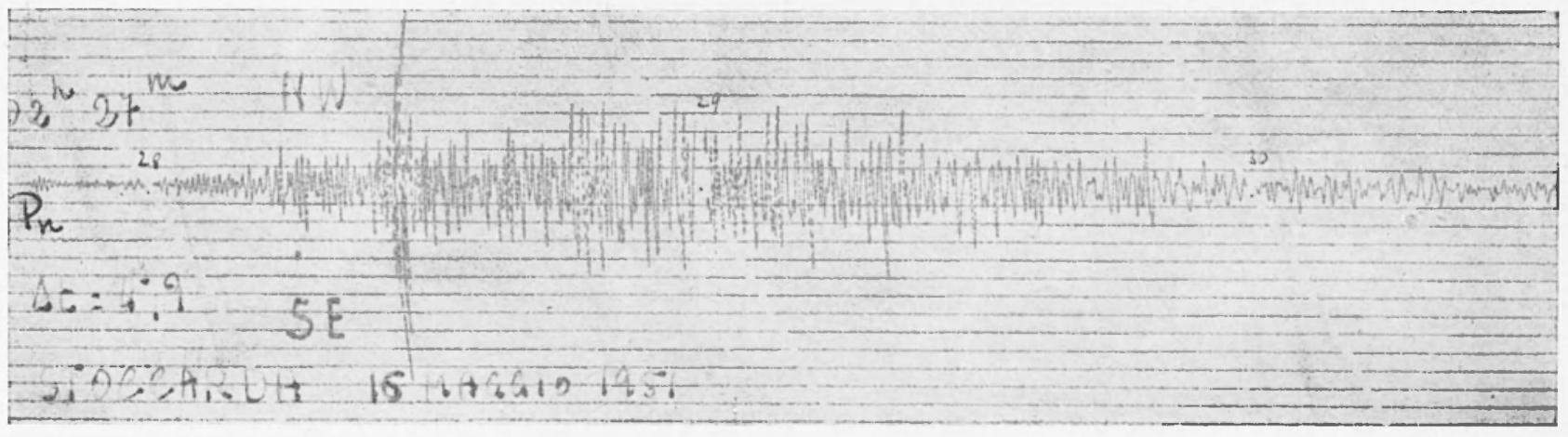

Fig. 19 d) - Stoccarda - II scossa. 

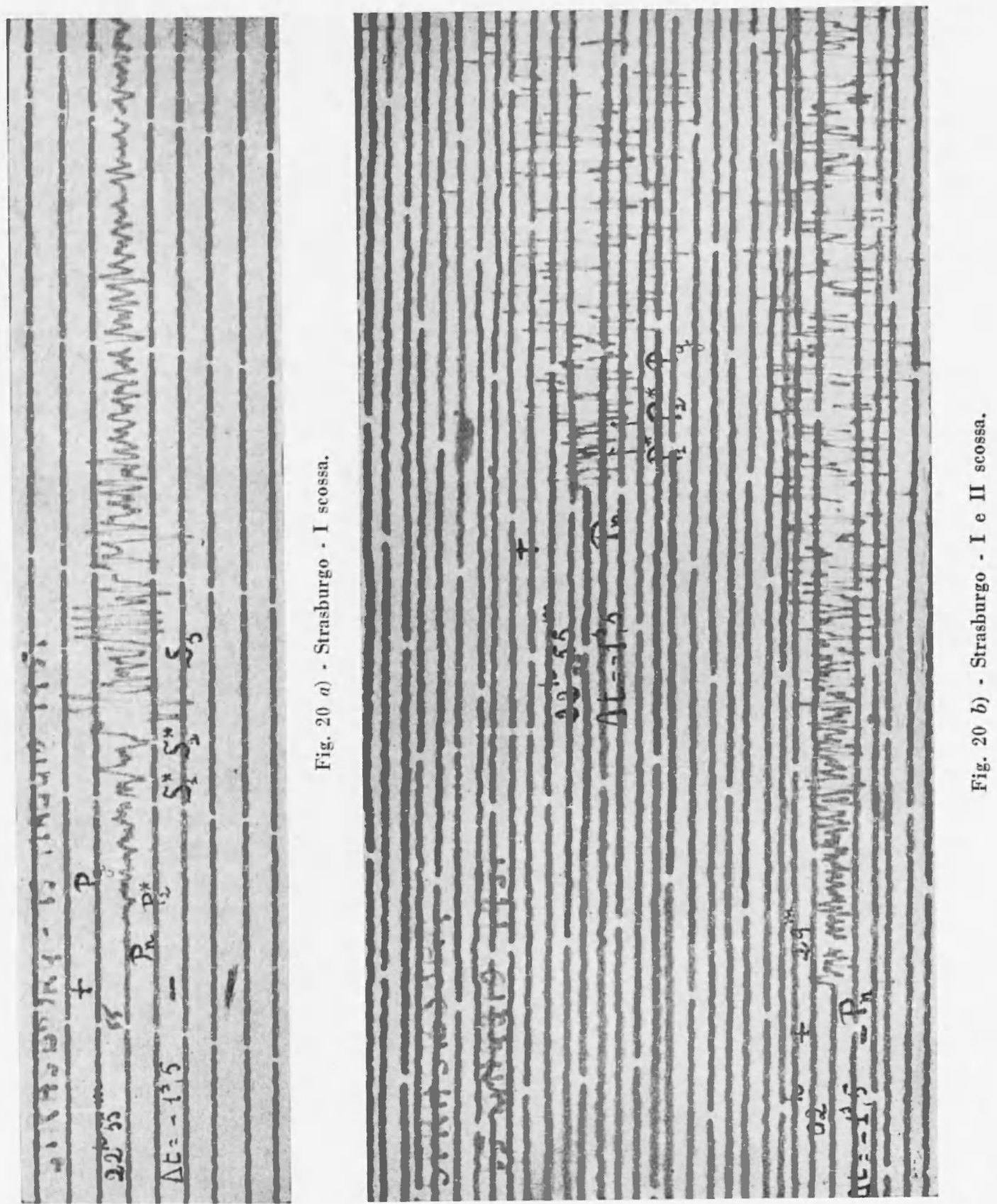


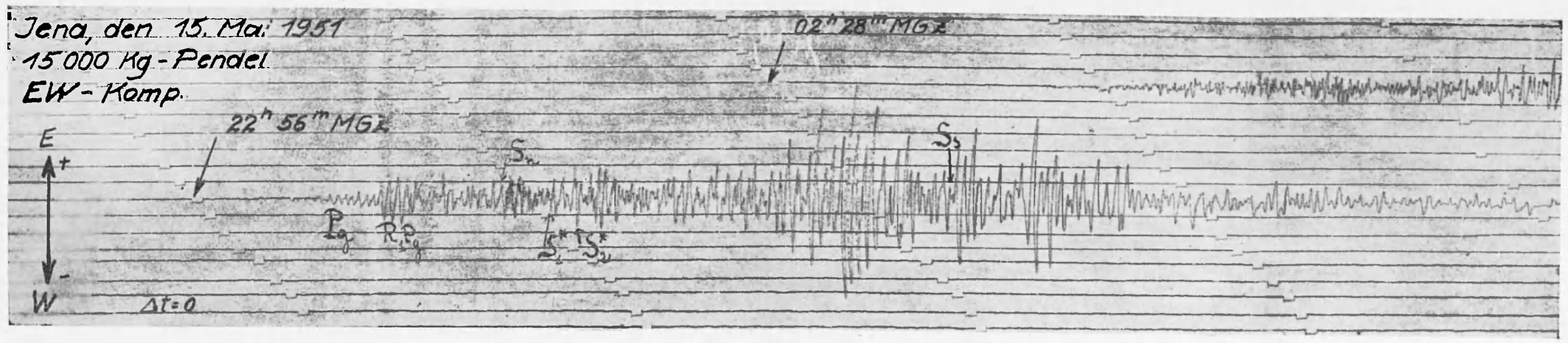

Fig. 21 a) - Jena - I scossa.

Jend,den 15 Mol 1951 $15000 \mathrm{~kg}$ - Pendel NS-Momp.

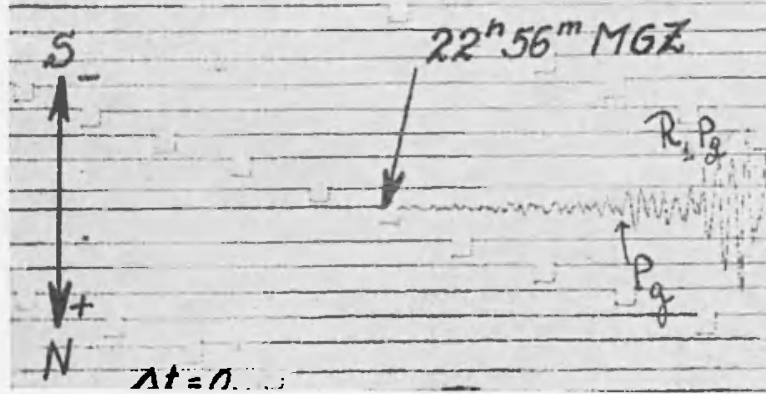

$$
02^{\mathrm{h} 28^{m}} 116 \mathrm{z}_{2}
$$

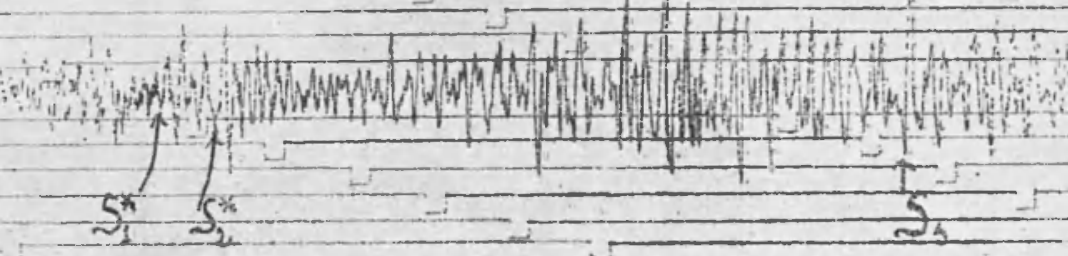

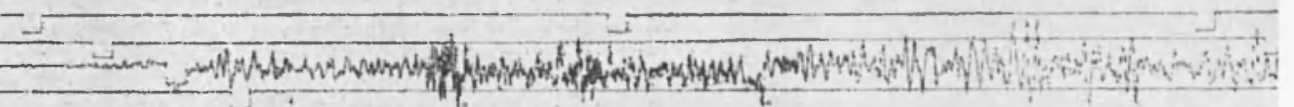

Fig. 21 b) - Jena - I scossa.

Station 'Gottingen. Wiectert-17-to Pendel. N5 Komp 15. $\bar{V} .51 .22^{\ln } 55^{\mathrm{M}}$... G G G $+$
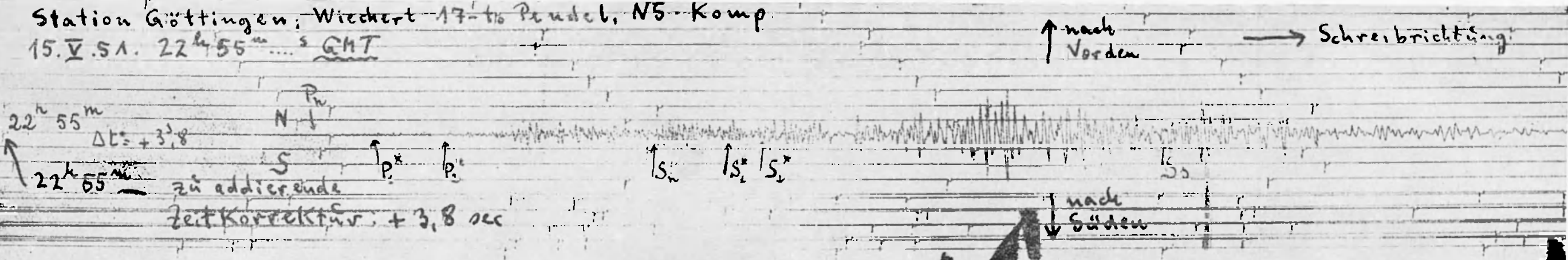

Fig. 22 - Gottinga - I scossa. 
c provocò oltre al crollo della torre di sondaggio, una serie di spaccature nel terreno circostante al soffione, lunghe molti metri, larghe una diecina di centimetri e con un sensibile dislivello fra gli orli di esso.

La popolazione di Basiasco fu costretta, per il pericolo di crolli, ad abbandonare temporaneamente le cose.

Dall'esame delle registrazioni sismiche ottenute presso l'Osservatorio geofisico di Pavia, si poté rilevare che il 5 marzo 1949 alle 18,30 ca., si era verificato un leggerissimo movimento tellurico, con un epicentro ad una distanza di circa $25 \mathrm{~km}$ da Pavia. Nei successivi mesi di aprile e maggio furono registrate presso il medesimo osservatorio ancora una trentina di altre piccole scosse, tutte con distanza epicentrale del medesimo ordine di grandezza di quella registrata il 5 marzo. Tali scosse possono essere senz'altro collegate alla violenta eruzione di gas di cui è detto sopra. Se eruzioni del genere possono verificarsi in profondità, è chiaro che l'energia sviluppata viene direttamente trasmessa al mezzo circostante, provocando movimenti sismici di intensità ben maggiore.

Roma - Istituto Nazionale di Geofisica - Dicembre 1954.

\section{RIASSUNTO}

Si espongono i risultati di un lungo lavoro, condotto con metodi diversi, sulle scosse sismiche che hanno colpito il Lodigiano il 15-16 maggio del 1951.

La scossa principale (quella del 15 maggio) ebbe il suo epicentro nei pressi di Caviaga, alla profondità di $5 \mathrm{~km}$ circa. Epicentro analogo risulta aver avuto la scossa del giorno successivo.

Lo studio dei vari tipi di onde sismiche, destate dalle scosse, ha portato ai seguenti valori per le velocità: onde $\mathrm{Pg}-5, \mathrm{l}$ km sec; $\mathrm{Pn}-8,16 ; \mathrm{Sg}-3,1 ; \mathrm{Sn}-4,52$. Per le onde $\mathrm{P}^{*}$ (o $\left.\mathrm{P}_{\mathrm{b}}\right)$ non è stato pos. sibile determinare una dromocrona unica. Un esame accurato delle re. gistrazioni, ha provato l'esistenza di due tipi d'onda, interessanti due diverse stratificasioni profonde, seguenti quella del "granito". Le ve. locità di queste onde - indicate con $i$ simboli $\mathrm{P}^{*}{ }_{2}$ e $\mathrm{P}^{*}{ }_{1}$ rispettivamente - sono risultate così espresse: $\mathrm{P}_{2}{ }_{2}-6,1 \mathrm{~km} / \mathrm{sec} \cdot \mathrm{P}_{1}{ }_{1}-6,9 \mathrm{~km} / \mathrm{sec}$. Almeno in corrispondenza della Val Padana, la crosta terrestre consiste quindi di tre strati sovrapposti, caratterizzati da velocità per le onde longitudinali di 5,$1 ; 6,1$ e $6,9 \mathrm{~km} / \mathrm{sec}$ rispettivamente. Sopra lo strato del "granito", si trova una stratificazione di sedimenti, generalmente 
diffusa in tutta Europa: nei sedimenti più consolidati, le onde longitudinali presentano velocità medie dell'ordine di 3,8-4,0 $\mathrm{km} / \mathrm{sec}$.

La determinazione della profondità ipocentrale e dello spessore delle stratificazioni ̀̀ stata molto laboriosa. La profondità ipocentrale è risultata di $5 \mathrm{~km}$ ca. Lo spessore medio della coltre alluvionale ha un valore di $6 \mathrm{~km}$ ca., mentre il successivo strato del "granito " presenta, uno spessore medio di $8-9 \mathrm{~km}$. Non riesce facile valutare lo spessore dei due strati «basaltici» seguenti: non dev'essere però tanto lontano dal vero un valore complessivo medio di $15 \mathrm{~km}$ circa.

Per quanto riguarda la natura della scossa all'ipocentro, la suddivisione superficiale delle compressioni e delle dilatazioni, suggerisce l'ipotesi di una violenta spinta verso l'esterno, secondo un angolo solido con asse fortemente inclinato verso NIW. La singolarità del meccanismo secondo cui la scossa si è determinata, il fatto che la zona interessata è notoriamente asismica e che in essa, da parecchi anni, è in corso un'abbondante estrazione di gas metano, ha fatto ritenere non del tutto improbabile che le scosse in esame siano comunque collegate allenorme decompressione in atto negli strati profondi, di dove il gas scaturisce con pressioni superiori ai $100 \mathrm{~kg} / \mathrm{cm}^{2}$.

\section{$S U M M A R Y$}

The following are the results of work carried out over a long period and employing different methods, on the seismic shocks which hit Lunigiano on the 15th-16th May, 1951.

The principal shoch (that of the 15th May) had its epicentre in the vicinity of Caviaga, at a depth of about $5 \mathrm{kms}$. The shock on the following day is reported to have the same epicentre.

The study of the various kinds of seismic waves to which the shochs gave rise has led to the following values for velocity: waves $\mathrm{Pg}=5,1$ $\mathrm{Km} /$ sec., $\mathrm{Pn}=8.16 ; \mathrm{S}_{\mathrm{g}}=3.1 ; \mathrm{Sn}=4.52$. For $\mathrm{P}$ (or $\mathrm{P}_{1}$ ) waves it was not possible to determine a single time-curve. A careful examination of the registrations proved the evistence of tyo types of waves, involving two different deep strata, following the "granite" one. The velocity of these waves - indicated bi the symbols $\mathrm{P}_{\text {.. }}$ and $\mathrm{P}_{1}$ respectively - were thus expressed: $\mathrm{P}_{2}=6.1 \mathrm{~km} / \mathrm{sec} ; \mathrm{P}_{1}=6,9 \mathrm{~km} / \mathrm{sec}$. The earth's crust, as regards the Val Padana at least, consists hence of there superimposed layers, characterized in its longitudinal waves by a velocity of 5.16 .1 and $6.9 \mathrm{~km} / \mathrm{sec}$ respectively. Overling the "granite» 
stratum is a stratification of sediments more or less common to all Europe. In the more solidified sediments, the longitudinal waves show average velocities ranging from $3,8-4,0 \mathrm{~km} / \mathrm{sec}$.

It was slow work rechoning the depts offocus and the thichness of the stratifications. The depth of focus proved to be about $5 \mathrm{~km}$. The average thickness of the alluvial layer comes to about $6 \mathrm{~km}$., while the following "granite" layer shows an average thichness of $8.9 \mathrm{~km}$. The thichness of the next two "Basalt" strata is not casy to establish: a total average figure of about $15 \mathrm{~km}$. however, should not be very far from the truth.

As regards the nature of the shock at the focus, the superficial subdivision of compressions and expansions suggests the idea of a violent outward thrust, in a solid angle with an axis strongly inclined tawards NIF. The uniqueness of the mechanism determining the shock, the fact that the zone concerned is notoriously aseismic and that from it, in recent years, methane gas has been extracted in large quantities, has made it not unlikely that the shocks there are in any case connected with the enormous decompression under way in the deep strata, whence the gas escapes with pressures exceeding $100 \mathrm{~kg} / \mathrm{cm}^{\circ}$.

\section{BIBLIOGRAFIA}

(1) Calor P., Nuovi metodi per la determinazione delle coordinate epicentrali e della profondità ipocentrale di un terremoto ad origine vicina. "La Ricerca scientifica "), $\mathrm{X}$, n. $7-8,1939$.

Calor P. Sulla determinazione delle coordinate spaziali di un terremoto ad erigine vicint. "Boll. Società Sismol. Ital. ", XXXVIII, 1940.

(2) Caloi P. Peronaci F. Il terremoto del Turkestan del 2 novembre 1946. "Annali di Geofisica », I, n. 2, 1948.

(3) Calor P., Sulla determinazione delle coordinate epicentrali di un terremoto ad origine vicina. "Atti Acc. Scienze di Torino ", 81 e 82, 1945-16, 1946-47.

(') Caloi P., Nuovo metodo per determinare le coordinate ipocentrali e la velocità di propagazione delle onde longitudinali e trasversali dirette. "Rendiconti R. Acc. d'Italia », serie VII, vol. IV, 1943.

Calor P., Caratteristiche sismiche Jondamentali dellEuropa centrale. "Boll. Società Sismol. Ital. ", XL, n. 34, 1942.

(5) Caloi P., Di Filippo D. Spanea M. C., Onde sismiche guidate dagli strati sodimentari. "Annali di Geofisica ", VIII, n. 1, 1955.

(6) Barate M., Terremoti d'Italia. Bocca, Torino, 1901.

(7) Baratra M., I terremoti in Italia. "Pubblicaz. Commissione Ist. Studio Grandi Calamità », VI, Le Monnier, 1936. 